ARTICLE

https://doi.org/10.1038/s41467-019-11892-5

\title{
GSNOR provides plant tolerance to iron toxicity via preventing iron-dependent nitrosative and oxidative cytotoxicity
}

\author{
Baohai Li (iD ${ }^{1,2,3,4}$, Li Sun ${ }^{5}$, Jianyan Huang ${ }^{2,6}$, Christian Göschl ${ }^{4}$, Weiming Shi ${ }^{5}$, Joanne Chory ${ }^{2,6}$ \& \\ Wolfgang Busch (1) 2,3,4
}

Iron $(\mathrm{Fe})$ is essential for life, but in excess can cause oxidative cytotoxicity through the generation of Fe-catalyzed reactive oxygen species. It is yet unknown which genes and mechanisms can provide Fe-toxicity tolerance. Here, we identify S-nitrosoglutathione-reductase (GSNOR) variants underlying a major quantitative locus for root tolerance to Fe-toxicity in Arabidopsis using genome-wide association studies and allelic complementation. These variants act largely through transcript level regulation. We further show that the elevated nitric oxide is essential for Fe-dependent redox toxicity. GSNOR maintains root meristem activity and prevents cell death via inhibiting Fe-dependent nitrosative and oxidative cytotoxicity. GSNOR is also required for root tolerance to Fe-toxicity throughout higher plants such as legumes and monocots, which exposes an opportunity to address crop production under high-Fe conditions using natural GSNOR variants. Overall, this study shows that genetic or chemical modulation of the nitric oxide pathway can broadly modify Fe-toxicity tolerance.

\footnotetext{
${ }^{1}$ College of Environmental and Resource Sciences, Zhejiang University, 866 Yuhangtang Road, Hangzhou, Zhejiang 310058, China. ${ }^{2}$ Plant Biology Laboratory, Salk Institute for Biological Studies, 10010 N Torrey Pines Rd, La Jolla, CA 92037, USA. ${ }^{3}$ Integrative Biology Laboratory, Salk Institute for Biological Studies, 10010 N Torrey Pines Rd, La Jolla, CA 92037, USA. ${ }^{4}$ Gregor Mendel Institute (GMI), Austrian Academy of Sciences, Vienna Biocenter (VBC), Dr Bohr-Gasse 3, 1030 Vienna, Austria. ${ }^{5}$ State Key Laboratory of Soil and Sustainable Agriculture, Institute of Soil Science, Chinese Academy of Sciences, No. 71 East Beijing Road, Nanjing 210008, China. ${ }^{6}$ Howard Hughes Medical Institute, Salk Institute for Biological Studies, La Jolla, CA 92037, USA. Correspondence and requests for materials should be addressed to B.L. (email: 0019054@zju.edu.cn) or to W.B. (email: wbusch@salk.edu)
} 
ron $(\mathrm{Fe})$ is an essential nutrient for every organism but can become a trigger to kill cells when present at elevated levels. In humans, Fe overload is correlated with many diseases such as those affecting vital organs like liver and heart, as well as neurodegenerative diseases including Alzheimer and early onset Parkinson ${ }^{1}$. In plants, serious symptoms of Fe toxicity have been recognized in rice for more than half a century ${ }^{2}$. In fact, $\mathrm{Fe}$ toxicity represents one of the most widely spread soil constraints for crop production in waterlogged soils, but also in a wide range of soil types including Ferralsols, Acrisols, Fluvisols, Podzols and Gleysols $^{3}$, and can cause up to $10-90 \%$ of yield loss in rice ${ }^{4,5}$. The Fe concentration causing Fe toxicity in rice ranges widely, from 10 to $>2000 \mathrm{mgL}^{-1}$ in the soil solution, and is highly dependent on other soil parameters such as geochemistry and nutrient levels, as well as on the particular rice variety ${ }^{6}$. It is estimated that $19 \%$ of the total area for rice production in Africa harbors a potential risk for $\mathrm{Fe}$ toxicity ${ }^{7}$. While engineering Fe toxicity tolerant rice varieties would be highly desirable, more than twenty attempts over the past two decades were not successful in identifying genes responsible for the variation of Fe toxicity tolerance in plants ${ }^{8-13}$

The toxicity of $\mathrm{Fe}$ is so far mainly attributed to its reaction with hydrogen peroxide $\left(\mathrm{H}_{2} \mathrm{O}_{2}\right)$ to generate the hydroxyl radical $\left(\mathrm{OH}^{*}\right)$ that is the most active reactive oxygen species (ROS) ${ }^{14-16}$. This reactive property of Fe can exert direct cellular damage via damaging biomolecules such as lipids or proteins, but also can regulate processes in growth and development. For instance, in humans, Femediated ROS is linked with many diseases ${ }^{1}$, but also required to kill cancer stem cells through the ferroptosis (a specific type of cell death $)^{17,18}$. In plants, Fe accumulation leads to primary root growth arrest induced by phosphorus deficiency and is associated with ROS over-generation $^{19}$. While Fe dependent ROS production is seen as the major mechanism for Fe toxicity, studies in yeast suggest that Fe-catalyzed ROS production may not account for Fe toxicity ${ }^{20}$. Similarly, knockout of UPB1, a transcription factor controlling ROS homeostasis in the primary root of Arabidopsis ${ }^{21}$, does not affect the sensitivity of the primary root to high levels $(500 \mu \mathrm{M})$ of $\mathrm{Fe}^{22}$. These results suggest that other components (additional to ROS) might be also required for Fe-mediated toxicity. On the other hand, ferritins (Fe binding proteins) seem to be the major components to buffer $\mathrm{Fe}$ overloading and consequently oxidative stress in both animals and plants ${ }^{23,24}$, but knockout of ferritin (triple mutant) does not change the sensitivity of the primary root in response to $500 \mu \mathrm{M} \mathrm{Fe} e^{22}$, which suggests the primary mechanism for plant root tolerance to Fe toxicity is still elusive. For all the extensive research conducted in the area of Fe biology and Fe toxicity in plants and animals and despite its broad relevance, not only the major genes responsible for high Fe tolerance have yet to be identified in plants, but also the ROS-independent mechanisms of Fe toxicity are not well understood. Here, we identify S-nitrosoglutathione reductase (GSNOR) as a major genetic regulator required for Fe toxicity resistance throughout higher plants and show that the nitric oxide (NO) pathway can be key for modulating tolerance to Fe toxicity.

\section{Results}

GWAS identify a locus linked to Fe toxicity. To identify genetic variants that confer plant tolerance to high $\mathrm{Fe}$, we made use of natural variation of primary root growth responses in Arabidopsis thaliana to high $\mathrm{Fe}$, as primary root growth is frequently used to evaluate heavy metal tolerance ${ }^{25}$ and also for high Fe toxicity in plants $^{22,26}$. We measured primary root length at high $\mathrm{Fe}$ and $\mathrm{Fe}$ tolerance (root length of high Fe relative to the control) among 319 natural accessions from day 3 to day 13 after germination in control $(1 \times \mathrm{MS})$ and high $\mathrm{Fe}(350 \mu \mathrm{M} \mathrm{Fe})$ conditions (Fig. 1a; Supplementary Fig. 1a, b). The broad sense heritability of the observed variation ranged from 0.442 to 0.523 in control conditions and from 0.411 to 0.492 in high Fe conditions, with the highest value observed for day 10 (Supplementary Table 1). We then performed genome-wide association studies (GWAS) and found only one significant peak associated with root length of high $\mathrm{Fe}$ and Fe tolerance that exceeded the Bonferroni-corrected significance threshold of 5\% (Fig. 1b, c; Supplementary Fig. 2a and $b$ ). This peak was located on chromosome 5 around the most significant single nucleotide polymorphism (SNP) at position 17684110 and was detected at multiple time-points (Fig. 1b, c; Supplementary Fig. 2b). Corroborating this peak, we also found this association using an alternative multi-trait GWAS approach (Supplementary Fig. 2c). As the SNP peak was the same regardless of using the absolute root length or relative root length at high $\mathrm{Fe}$, or the multi-trait GWAS, we decided to use the absolute root length at high Fe for further analysis. Moreover, we chose to focus on the day 10 GWAS as the representative GWAS, as the broad heritability was highest at this day in control and high Fe conditions (Supplementary Table 1). We then analyzed the associated peak further and found eight significantly associated SNPs covering several genes (Fig. 1d, e). All of these SNPs were highly linked (Supplementary Fig. 3a). When conducting a conditional GWAS using the lead SNP:17684110, we found that only a single SNP still exceeded the Bonferroni-corrected threshold (Supplementary Fig. 3b). This genetic locus explained a notable proportion $(20 \%)$ of the root growth variation at high Fe. The T-variant (54\%) of this lead SNP was associated with higher Fe tolerance and the A-variant (46\%) was associated with lower Fe tolerance within these 319 accessions (Supplementary Fig. $3 \mathrm{c}$ ). Overall, this suggested that a genetic locus in that region is responsible for a major fraction of root growth variation among these 319 accessions upon high Fe.

Natural allelic variation of GSNOR underlies Fe tolerance. To identify the causal gene for Fe tolerance within this genomic region, we first analyzed the gene expression of all ten genes surrounding the lead SNP in a publicly available data set (https:// www.ebi.ac.uk/arrayexpress/experiments/E-GEOD-53197) containing both root and shoot expression data of 17 Arabidopsis accessions (Supplementary Fig. 3d, e). We reasoned that a causal gene might be differentially expressed already in control conditions in Arabidopsis accessions containing different lead SNP variants. Indeed, we found one gene, AT5G43940, which encodes a S-nitrosoglutathione reductase (GSNOR) and displayed a supporting expression pattern: it was significantly different between T-variant accessions (higher GSNOR expression) and A-variant accessions (lower GSNOR expression) in both roots and shoots under control conditions (Supplementary Fig. 3d, e). These results suggested that GSNOR might be the causal gene and expression variation of GSNOR might be responsible for the natural variation of root tolerance to high Fe.

To explore the pattern of natural variation of GSNOR transcript in a larger population, we analyzed the expression variation of GSNOR in shoots of 665 Arabidopsis accessions ${ }^{27}$ and found that its expression varied notably between different accessions (Supplementary Fig. 3f). We then performed an expression quantitative trait loci (eQTLs) mapping using GWAS with the GSNOR expression in these 665 accessions. Interestingly, we found a single significant SNP peak in immediate proximity to the GSNOR locus (Fig. 2a-c). These results suggested that the variation of GSNOR expression in the shoot can be caused by cisregulatory variants. As there was a strong correlation between root and shoot expression of GSNOR of 17 accessions for which expression data was available (Pearson correlation coefficient is $0.599, p$-value $=0.011)$, this hinted towards the relevance of cis- 
a
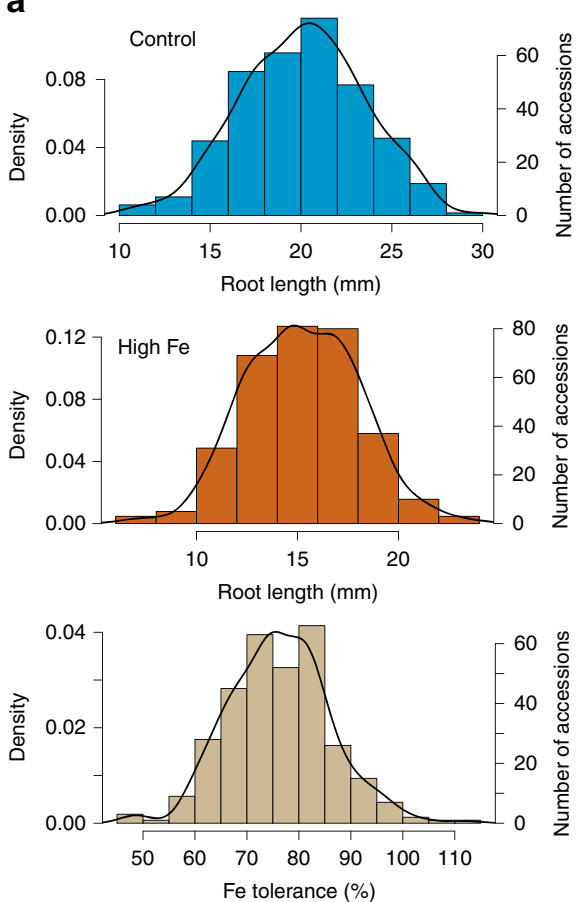

C

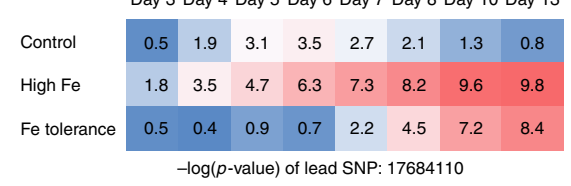

b
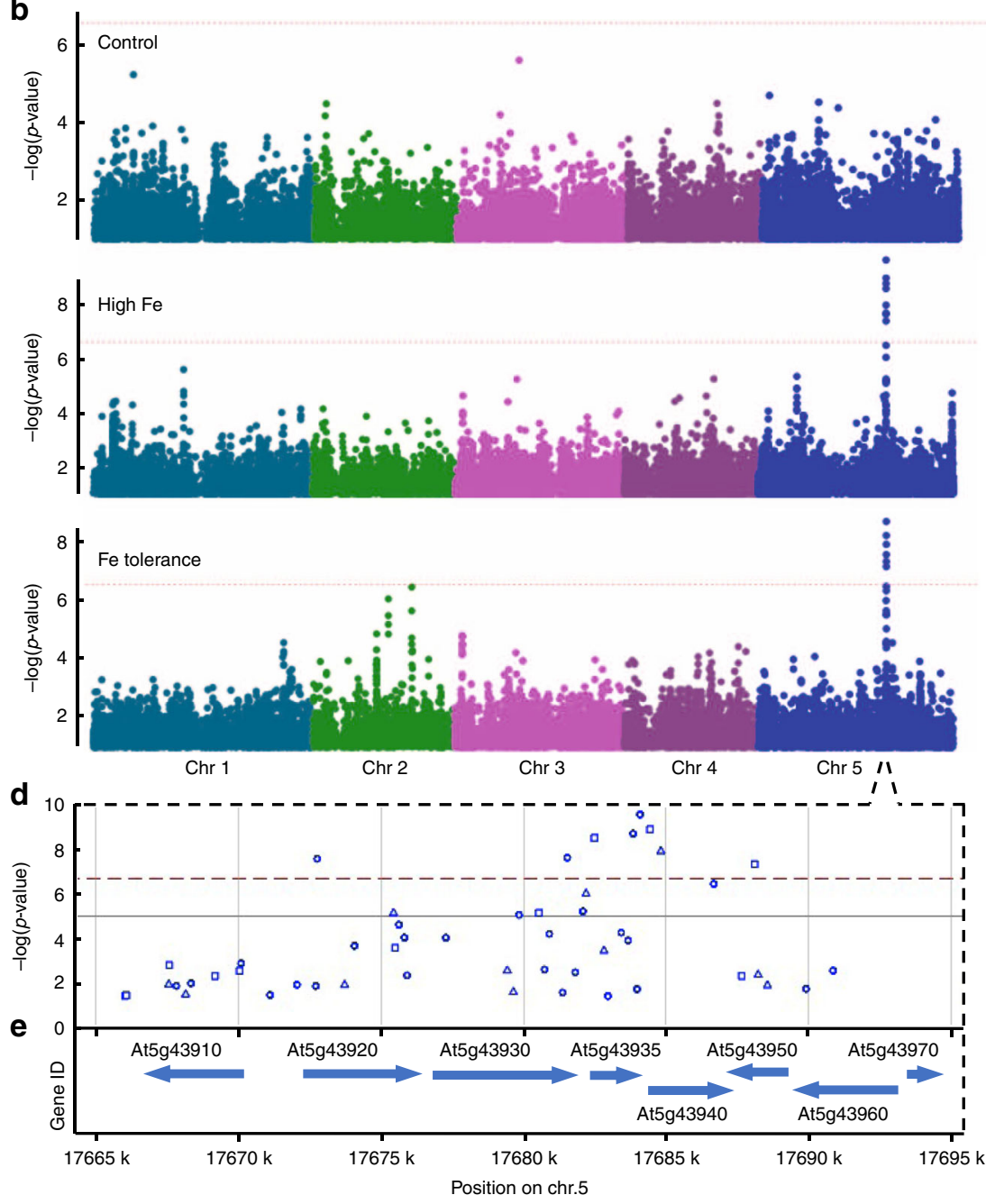

Fig. 1 GWAS identifies GSNOR as associated with the root growth response to high Fe. a Distributions of root length and Fe tolerance index in 319 accessions at day 10. b Manhattan plots of GWAS using traits shown in a. Different colors indicate five chromosomes. The horizontal dashed lines indicated the thresholds of significance. $\mathbf{c}$ A heatmap of $\log (p$-value) of lead SNP identified at high Fe in $\mathbf{b}$ over the course of time. $\mathbf{d}$, e Regional plots of the significant SNPs $\mathbf{d}$ and genes $\mathbf{e}$ identified at high Fe in $\mathbf{b}$. GSNOR is encoded by At5g43940. Circle, triangle, and square denote the SNP to cause intergenic, non-synonymous_coding, and synonymous_coding changes, respectively. The source data of Fig. 1a are provided in a Source Data file

regulatory elements for the causality of the natural variation of root growth responses to high $\mathrm{Fe}$.

Consistent with our hypothesis that GSNOR is involved in $\mathrm{Fe}$ tolerance, root growth of both gsnor knockout mutants (hot5-2 in Col-0 background and hot5-4 in Ws- 4 background) ${ }^{28}$ was very sensitive to high $\mathrm{Fe}$ (above $150 \mu \mathrm{M} \mathrm{Fe}$ ) (Fig. 3a, b) and both mutant lines showed no root growth at $350 \mu \mathrm{M} \mathrm{Fe}$ in $1 / 2 \times \mathrm{MS}$ agar medium. The observed sensitivity to high Fe was not restricted to root growth but encompassed traits in the whole seedling as the leaves of gsnor knockout mutants were much smaller than the wild-type at $250 \mu \mathrm{M} \mathrm{Fe}$ (Fig. 3a). The roots of hot5-2 mutants displayed $89 \%$ growth inhibition when $\mathrm{Fe}$ concentration increased from $50 \mu \mathrm{M}$ to $250 \mu \mathrm{M}$, while only $20 \%$ inhibition was observed in wild-type roots at day 6 (Fig. 3b). $150 \mu \mathrm{M} \mathrm{Fe}$ already inhibited $70 \%$ of root growth in hot5-2 mutants compared to the control, while it only caused $17 \%$ inhibition in wild-type at day 6 (Fig. 3b). Therefore, we considered the concentration of Fe at/above $150 \mu \mathrm{M}$ in $1 / 2 \times$ MS agar medium as high Fe in our subsequent experiments. Complementation of hot5-2 using a pGSNOR:GSNOR-GFP transgene ${ }^{29}$ completely recovered the root growth of hot5-2 at both $150 \mu \mathrm{M}$ and $250 \mu \mathrm{M}$ of Fe (Fig. 3b). Ubiquitous overexpression of GSNOR ${ }^{30}$ in Col-0 using the $35 \mathrm{~S}$ promoter (Supplementary Fig. 14) slightly enhanced tolerance to $150 \mu \mathrm{M}$ Fe (Fig. 3b). Taken together, this demonstrated that GSNOR is required for root growth tolerance to high $\mathrm{Fe}$, but that its ubiquitous overexpression in a tolerant accession does not necessarily lead to strong gains in Fe tolerance.

Next, we wanted to test whether natural GSNOR variants are causal for the variation of high $\mathrm{Fe}$ tolerance. We therefore conducted an analysis of the polymorphism patterns that distinguish $\mathrm{Fe}$ tolerant and sensitive accessions. When grouping accessions according to their $\mathrm{Fe}$-sensitive $\mathrm{A}$-variant and $\mathrm{Fe}$ tolerant T-variant of the lead SNP and then analyzing their full genome sequences at the GSNOR locus, we found ample amount of sequence variation that distinguishes these two groups (Supplementary Fig. 4). The vast majority of this variation is non-coding and only one non-synonymous mutation in the amino acid sequence of GSNOR is strongly associated with the Avariant group of accessions (Supplementary Fig. 4). The complex pattern of polymorphisms within the A-variant group suggested that multiple alleles of GSNOR might contribute to the shorter roots in high $\mathrm{Fe}$ conditions. We therefore performed a haplotype analysis of the GSNOR sequence including the promoter region using the significant 8 SNPs identified by GWAS and found 10 


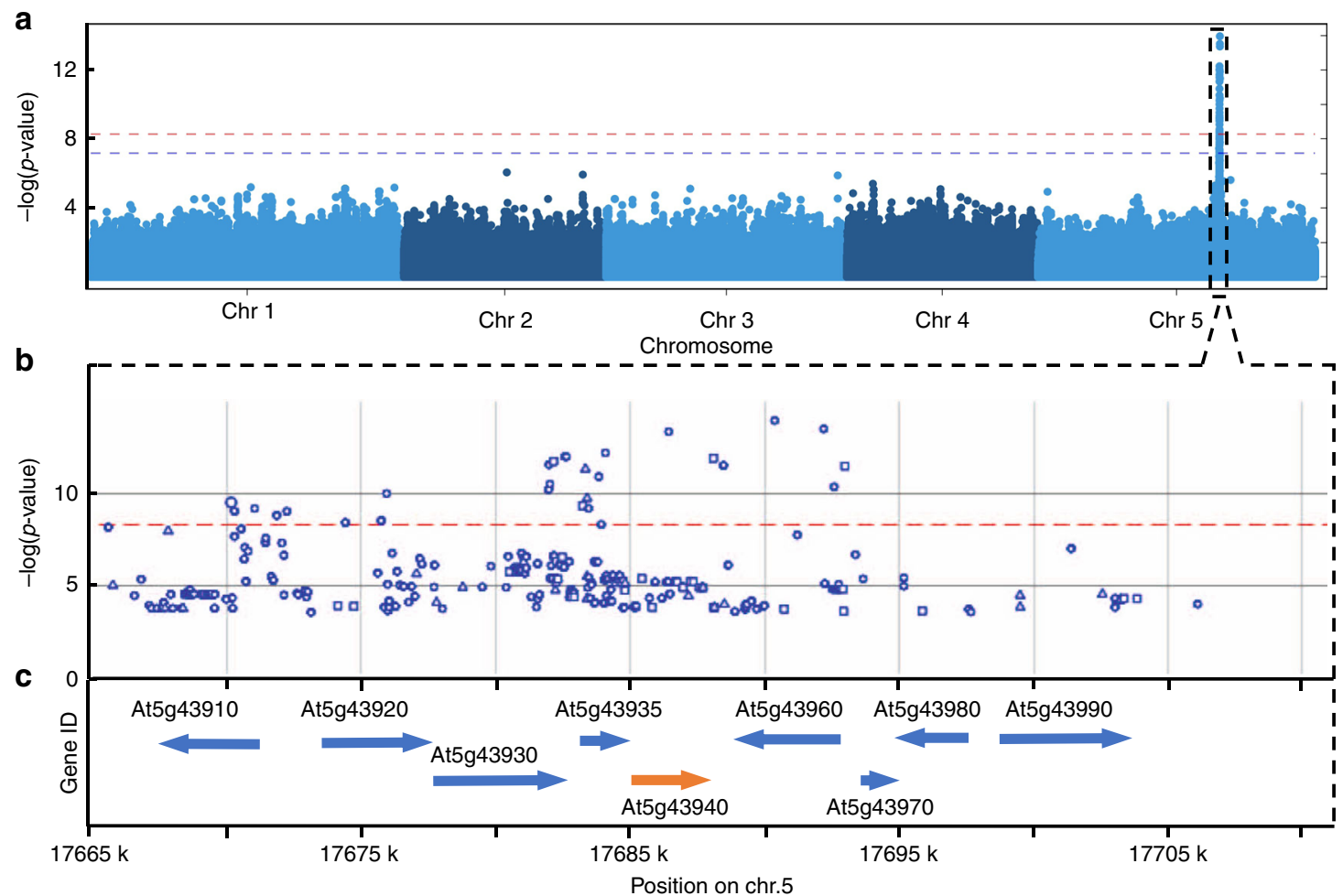

Fig. 2 Natural GSNOR variants go along with transcription level variation. a Manhattan plots of GSNOR eQTL mapping of 665 Arabidopsis accessions using GWAS analysis. The expression levels of GSNOR gene are depicted in Supplementary Fig. $2 \mathrm{f}$. b, $\mathbf{c}$ Regional plots of the significant SNPs $\mathbf{b}$ and gene models c. GSNOR is encoded by At5g43940. Circle, triangle, and square denote the SNP to cause intergenic, non-synonymous_coding, and synonymous_coding changes, respectively

haplotypes (at least present in 10 accessions) in the 319 accessions. Haplotype A, the biggest group, was found in 109 accessions and covers all variants of these 8 SNPs associated with high Fe sensitivity, while the second largest group, haplotype J, was found in 81 accessions and contains all variants of these 8 SNPs associated with high Fe tolerance (Supplementary Fig. 5). Based on root growth in high $\mathrm{Fe}$, haplotype A is not significantly different from haplotype $\mathrm{B}$ and $\mathrm{C}$, but is significantly different from the other haplotypes (Supplementary Fig. 5). These two biggest groups (haplotype $\mathrm{A}$ and J) defined by the haplotype analysis are essentially similar to the two groups defined by the two variants of the lead SNP. Therefore, we chose GSNOR from Col-0 (GSNOR_Col-0) in haplotype J to represent the Fe tolerant variant, while we chose GSNOR from Sf-2 (GSNOR_Sf-2) in haplotype $\mathrm{A}$ to represent the sensitive variant for further functional analysis.

To experimentally test whether GSNOR variants of Col-0 (GSNOR_Col-0) and Sf-2 (GSNOR_Sf-2) lead to differences in Fe tolerance, we transformed these two GSNOR variants (encompassing 826 bp upstream of the translational start codon, the GSNOR gene and 166-bp downstream of the translational stop codon based on the Col-0 reference genome) into the hot5-4 mutant (Fig. 4a), which has a better seed production than the hot5-2 mutant. Consistent with the result of the haplotype analysis, the average root growth of all independent GSNOR_Col-0 variant T2 lines showed significantly more tolerance to high $\mathrm{Fe}$ than all respective GSNOR_Sf-2 variant lines (Fig. 4b, c). Similar differences were again observed in two T3 lines for each GSNOR variant (Fig. 5a). The high Fe root growth tolerance conferred by the GSNOR_Col-0 alleles also correlated with an approximately two-fold increase in shoot biomass under high $\mathrm{Fe}$ compared to the sensitive allele that we measured in the T3 lines (Fig. 5b), demonstrating the relevance of GSNOR dependent high Fe tolerance at the organismal level.
In line with our observation of increased GSNOR transcript levels in Fe-tolerant accessions, the GSNOR_Col-0 variant conferred a significantly higher GSNOR mRNA expression as well as protein level (Fig. 5c, d) and enzyme activity level (Supplementary Fig. 6c) than the GSNOR_Sf-2 variant. Interestingly, the expression of GSNOR from both, a high Fe resistant variant (GSNOR_Col-0) and a high $\mathrm{Fe}$ sensitive variant (GSNOR_Sf-2), was not further induced by high Fe treatment (Fig. 5c), suggesting that the steady-state expression of GSNOR might be key for the increased Fe tolerance. Further support for the importance of GSNOR expression level for the observed effects of GSNOR natural variants came from the independently derived T3 lines harboring identical GSNOR sequences. Some of these independently derived GSNOR_Sf-2 lines displayed different levels of GSNOR expression, presumably due to their T-DNA insertion site. As we measured not only expression level but also root growth in these lines, we could therefore analyze whether there is a relation between these two variables. Indeed, line 2, the most Fe tolerant line, displayed a significantly higher GSNOR transcript level than line 4 and line 9, which were significantly more sensitive to high Fe (Supplementary Fig. 6a, b).

Collectively, these results show that natural GSNOR variants cause variation of Fe toxicity tolerance and that this is most likely due to SNPs that cause GSNOR expression level variation.

GSNOR counteracts Fe-dependent $\mathrm{NO}$ and $\mathrm{H}_{2} \mathrm{O}_{2}$ toxicity. As GSNOR activity confers Fe tolerance, we set out to explore the underlying molecular mechanism. GSNOR degrades Snitrosoglutathione (GSNO) that is formed by the reaction of nitric oxide (NO) and glutathione $(\mathrm{GSH})^{31,32}$ and thereby regulates NO signaling 28,33,34 (Supplementary Fig. 7a). Previous studies had shown that both, NO and GSH are highly 

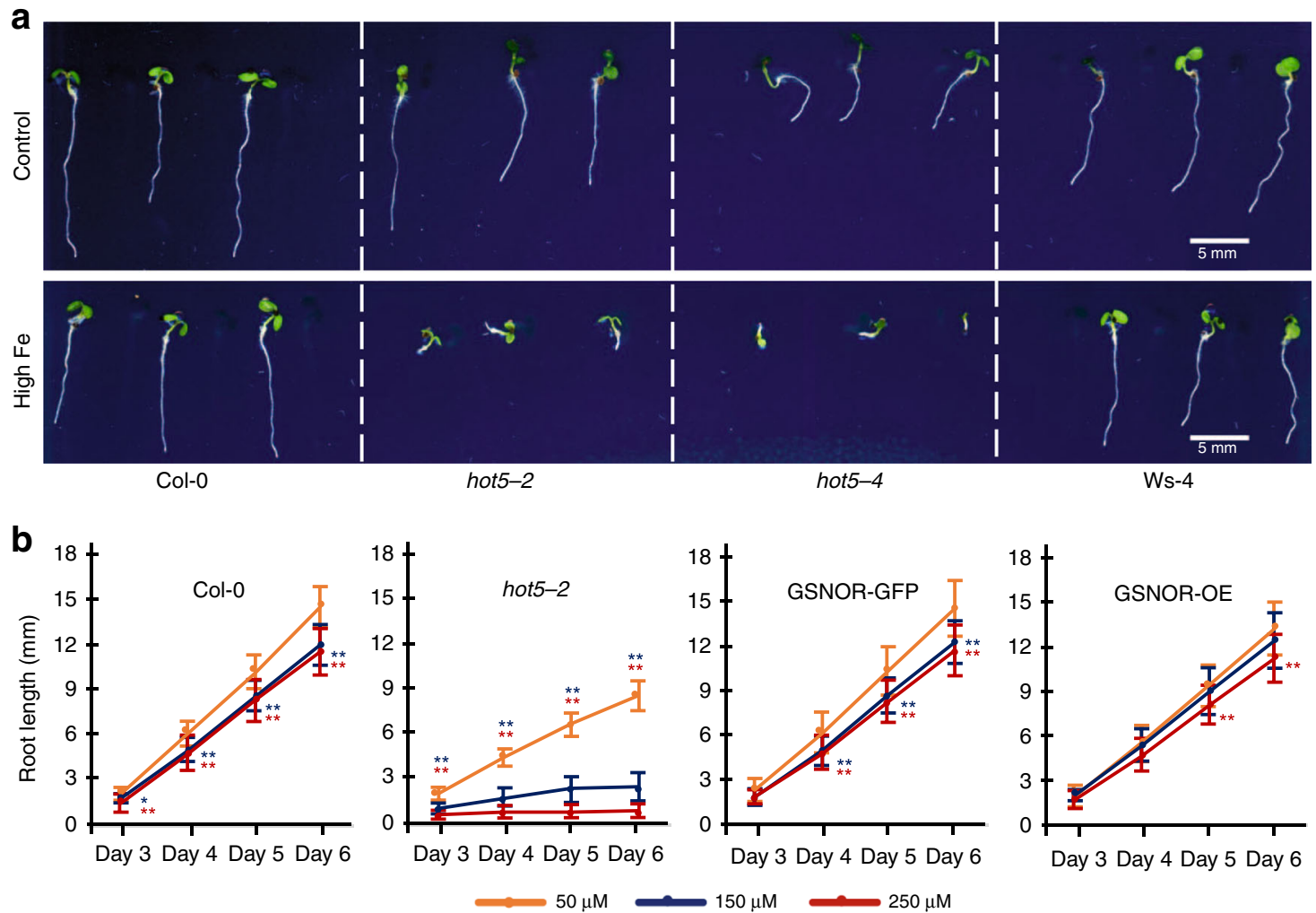

Fig. 3 GSNOR is required for root tolerance to high Fe. a Seedlings of gsnor knockout mutants (hot5-2 and hot5-4) and the respective wild-type (Col-0 and Ws-4) upon high Fe $(250 \mu \mathrm{M})$ treatment for 6 days. Scale bar, $5 \mathrm{~mm}$. b Mean root length of wild-type (Col-0), gsnor mutant (hot5-2), complemented line (GSNOR-GFP), and over-expression (GSNOR-OE) line. Error bars: standard deviation. $n=15$ biologically independent samples. Asterisk * and ** represent the significant differences between high Fe $(150 \mu \mathrm{M}$ Fe in blue asterisk, $250 \mu \mathrm{M}$ Fe in red asterisk) and the control as determined by Student's $t$-test at $p$-value $<0.05$ and 0.01 , respectively. The source data of Fig. $3 \mathrm{~b}$ are provided in a Source Data file

accumulated in the GSNOR knockout mutants ${ }^{35}$. We therefore first tested whether the accumulation of GSH arrests root growth in gsnor mutants at high Fe. For this, we analyzed the effect of GSH and L-Buthonine-sulfoximine (BSO, an inhibitor of gammaglutamylcysteine synthetase, consequently reducing GSH synthesis) on the root growth response to high $\mathrm{Fe}$. We found that root growth in gsnor mutants was decreased in high $\mathrm{Fe}$ when depleted of GSH (via treatment with BSO), but was not significantly changed by application of $0.5 \mathrm{mM}$ GSH (Supplementary Fig. 7b). Overall, these results did not support a model in which internal GSH accumulation is the key factor to cause the highly Fe sensitive phenotype in the gsnor mutant.

We next tested the contribution of the NO accumulation to the highly Fe sensitive phenotype in gsnor mutants. Application of 30 $\mu \mathrm{M}$ or $60 \mu \mathrm{M}$ sodium nitroprusside (a NO donor) significantly reduced root length in wild-type (37.4\% and $39.2 \%)$, gsnor mutant $(65.4 \%$ and $75.1 \%)$ and the complemented line $(36.6 \%$ and $30.1 \%$ ) at a level of $150 \mu \mathrm{M} \mathrm{Fe}$, respectively (Fig. 6a). This showed that high levels of NO can cause gsnor mutants to be more sensitive to high Fe. We further tested the contribution of NO to Fe toxicity, by assessing root growth in another mutant $C A B$ Underexpressed 1/ Nitrous Oxide Overexpressor 1, cue1 that is known to accumulate high levels of NO in the root tip ${ }^{36,37}$. Again, root growth in the cue1-6 mutant was more sensitive to high Fe compared to the wild-type (Supplementary Fig. 7c). Moreover, the application of cPTIO, a NO scavenger, significantly improved the root growth rate in gsnor mutants (2.9 fold) compared to wild-type (1.2 fold) at $150 \mu \mathrm{M}$ Fe (Supplementary Fig. 7d). Consistent with the contribution of NO to Fe toxicity, we found that high Fe elevates NO levels in the root meristem, which mimics the phenotype of $\mathrm{NO}$ accumulation in gsnor mutants that display this elevated NO level already in control conditions (Fig. $6 \mathrm{~b})$. Consistent with the regulatory role in NO homeostasis, GSNOR is highly expressed in the root tip particularly in the meristem zone ${ }^{29}$ (Fig. 6c). Collectively, these results revealed that NO accumulation in gsnor mutants impairs root tolerance to high $\mathrm{Fe}$ and that genetical or chemical modulation of NO levels changes the tolerance of root growth to high $\mathrm{Fe}$.

In contrast to the hyper-sensitivity at high $\mathrm{Fe}$, root growth of the gsnor mutant was similar to the wild-type when grown on high NO but at a decreased Fe concentration of $5 \mu \mathrm{M}$ (Fig. 6a). This suggested that the contribution of GSNOR in NO-mediated nitrosative inhibition of root growth depends on the $\mathrm{Fe}$ availability. We therefore hypothesized that Fe accumulation in the root tip may be one of the consequences of elevated internal NO level in the gsnor mutant, and thereby contributes to the reduced root growth rate. Consistent with this hypothesis, using Perl's DAB staining, we found that Fe accumulation in the root tip of gsnor mutants was higher than the wild-type grown in control condition $(50 \mu \mathrm{M} \mathrm{Fe})$. However, this accumulation was not observed at lower Fe levels $(10 \mu \mathrm{M} \mathrm{Fe})$ in the medium (Supplementary Fig. 8a). This suggests that $\mathrm{Fe}$ accumulated in gsnor mutants largely depends on externally available $\mathrm{Fe}$ or apoplastic Fe. Consistently, reduction of Fe levels from the control to low Fe could further rescue the root growth of gsnor mutant (Fig. 6a; Supplementary Fig. 8b). Collectively, these data show that the protective role of GSNOR in nitrosative stress on root growth is highly correlated with $\mathrm{Fe}$ availability in the root tip.

The generation of Fe-dependent ROS is considered to be the major reason for Fe toxicity ${ }^{14-16}$. Therefore, GSNOR may be 
a

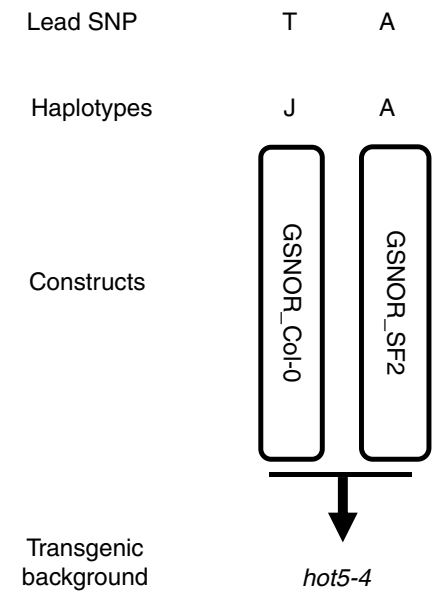

C

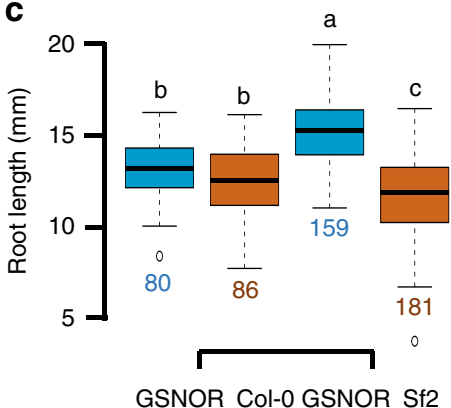

b
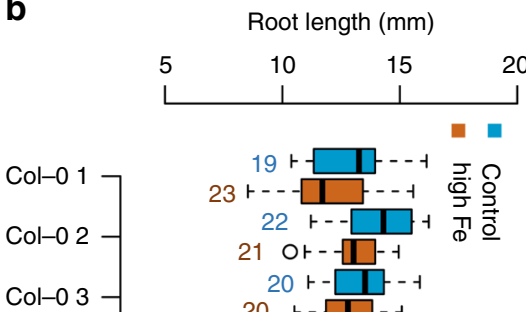

$20+-\square-1$
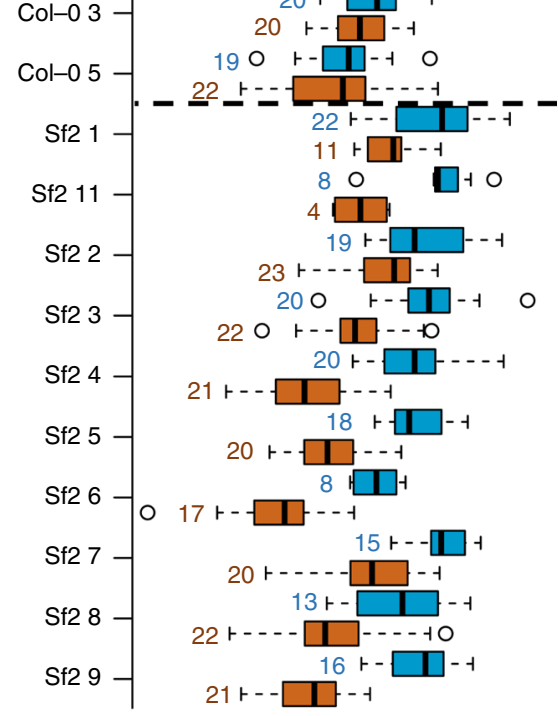

Ws-4 -

Fig. 4 Natural GSNOR variants cause variation of root growth tolerance to high Fe. a Schematic of the constructs for GSNOR variants to generate transgenic lines. b Box plots for root length of T2 seedlings of ten GSNOR_Sf-2 lines and four GSNOR_Col-0 lines at day 6. hot5-4 and Ws-4 were included as the comparison for high Fe sensitivity. Not detected (nd) indicates no root growth of hot $5-4$ was observed at $350 \mu \mathrm{M}$ Fe conditions. c Box plots for the mean of ten GSNOR_Sf-2 lines and four GSNOR_Col-O lines shown in $\mathbf{b}$. Numbers below and next to boxes in $\mathbf{b}, \mathbf{c}$ indicate the number ( $n$ ) of T2 seedlings used for each line in the analysis. Different letters indicate significant difference according to Tukey's HSD comparison $(p$-value $<0.05)$. The Source data of Fig. 4b are provided in a Source Data file

required for avoiding not only nitrosative stress but also oxidative stress. We therefore tested the root growth of gsnor mutant in response to exogenous $\mathrm{H}_{2} \mathrm{O}_{2}$, and found that root growth in gsnor mutant plants is very sensitive to $\mathrm{H}_{2} \mathrm{O}_{2}$ (Supplementary Fig. 8c and d). Moreover, gsnor hyper-sensitivity to $\mathrm{H}_{2} \mathrm{O}_{2}$ could be also alleviated by reducing $\mathrm{Fe}$ concentration in the medium (Supplementary Fig. 8c, d). Hence, similar to nitrosative stress, this result suggests that high $\mathrm{Fe}$ enhances $\mathrm{H}_{2} \mathrm{O}_{2}$ damage in gsnor mutants. Moreover, the protective role of GSNOR may be specific to $\mathrm{Fe}$-dependent $\mathrm{H}_{2} \mathrm{O}_{2}$-mediated oxidative toxicity, as the gsnor shoots and roots were tolerant to paraquat ${ }^{34}$ (Supplementary Fig. $8 \mathrm{e})$, another common oxidative stress inducer and converting oxygen $\left(\mathrm{O}_{2}\right)$ to the superoxide $\left(\mathrm{O}_{2}{ }^{-}\right)$radical ${ }^{38}$.

The root meristem is the center of cell proliferation and differentiation and thereby central for determining root growth ${ }^{21}$. We therefore examined the size of root meristem at different $\mathrm{Fe}$ levels and found that root meristem size in gsnor mutants was strongly decreased in high Fe conditions compared to wild-type (Fig. 6d, e). At $350 \mu \mathrm{M} \mathrm{Fe}$ in the medium, the root tips of gsnor mutants displayed cellular disorganization and radial swelling of cells in the outer tissue layers (Fig. 6d). At $50 \mu \mathrm{M} \mathrm{Fe,} \mathrm{gsnor}$ mutant root meristems were still dramatically shorter than the wild-type (Fig. 6e) and only at $5 \mu \mathrm{M} \mathrm{Fe}$, root meristem morphology and length recovered to that of wild-type plants (Fig. 6d, e). We also observed that high Fe caused a large number of dead cells in the gsnor root meristems using both propidium iodide (PI) and Sytox Orange staining (Fig. 6d; Supplementary Fig. 9). PI stains the walls of living plant cells but is also used as a marker for loss of membrane integrity and cell death, while Sytox Orange is a cell death marker ${ }^{39}$. Even at intermediate $\mathrm{Fe}$ levels $(150 \mu \mathrm{M} \mathrm{Fe})$ we observed frequent cell death in the root initials and their daughter cells in the meristem of gsnor mutant, but not in the wild-type (Supplementary Fig. 9). Moreover, we found that root meristem size, as well as cell death phenotypes in gsnor mutants were also very sensitive to $\mathrm{H}_{2} \mathrm{O}_{2}$, and depend on $\mathrm{Fe}$ availability (Fig. 6f, g). These results suggest that GSNOR determines the interaction of $\mathrm{NO}$ and Fe-dependent oxidative stress pathways to induce cell death in root meristems.

GSNOR is required for high Fe tolerance in higher plants. The important function of GSNOR in conferring tolerance to high Fe in Arabidopsis prompted us to test whether it is conserved in other plant species. GSNOR is present as a single copy in the genomes of most plant species including the graminaceous species rice $^{29}$ (Fig. 7a; Supplementary Fig. 10a). As high iron tolerance rice would be agronomically desirable in rice, we first chose to test the contribution of OsGSNOR in Fe toxicity tolerance in japonica rice (Oryza sativa) cultivar Zhonghua 11 (ZH11) by generating a knockout of OsGSNOR (Os02G57040) using the CRISPR/Cas9 system. Similar to Arabidopsis, three independent 
a
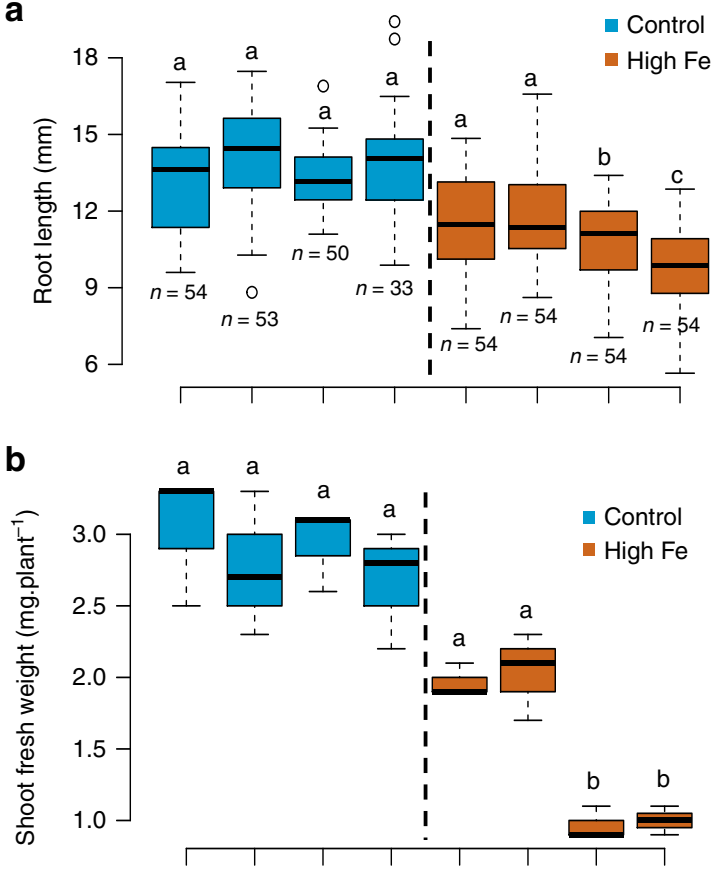

C
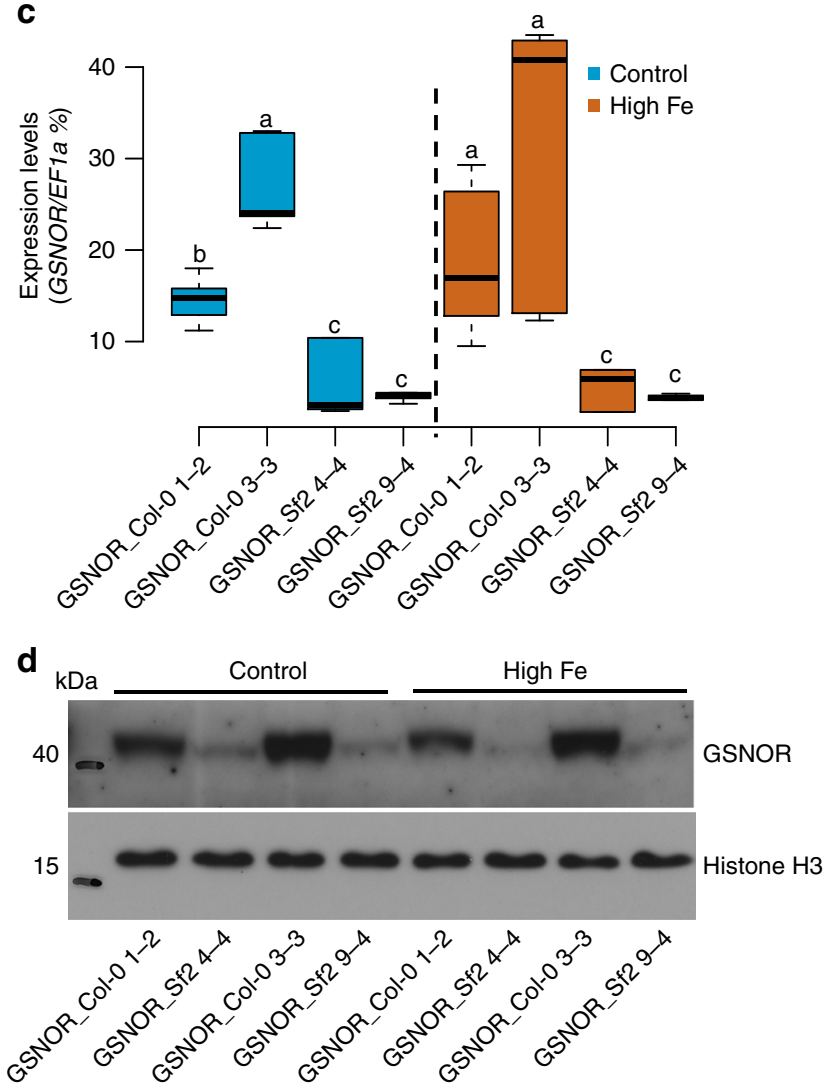

OsGSNOR knockout lines (Osgsnor-1, Osgsnor-2, and Osgsnor-3) containing frameshifts, displayed slightly reduced root growth under control conditions, but were dramatically more sensitive to high $\mathrm{Fe}$ (in hydroponics with $1000 \mu \mathrm{M} \mathrm{Fe}$ ) compared to the wildtype ZH11 (Fig. 7b, c; Supplementary Fig. 11a, b). At this early stage, shoot growth was also slightly retarded in these three OsGSNOR knockout lines in control conditions, and much more so in high Fe conditions (Supplementary Fig. 11c). The reduction of height in Osgsnor knockout lines compared to wild-type
Fig. 5 Natural GSNOR variants cause variation of GSNOR transcript levels and high Fe tolerance. a Box plots for root length of GSNOR_Col-O and GSNOR_Sf-2 transgenic T3 lines at control and high Fe (350 $\mu \mathrm{M} \mathrm{Fe})$ at day 6. $\mathrm{n}$, biologically independent samples. b Shoot biomass at day $15(n=3$ biologically independent samples). c GSNOR expression in roots at day 6 ( $n=6$ including 3 biologically independent samples and 2 technical replicates for each sample). The seedlings were T3 generation. Different letters indicate significant difference with Tukey's HSD comparison ( $p$ value $<0.05$ ) between different genotypes in a given condition. $\mathbf{d}$ Western blot analysis of GSNOR protein in 7-day-old seedlings at control and high Fe $(350 \mu \mathrm{M} \mathrm{Fe})$. Histone $\mathrm{H} 3$ protein is shown as the protein loading control. The source data are provided in a Source Data file

amounted to $12 \%$ when treated with high Fe for 2 weeks. Thus, OsGSNOR contributes to both, root and plant tolerance to $\mathrm{Fe}$ toxicity in rice.

Duplications of the GSNOR gene can be found in some plant species, and commonly occur in legumes including Lotus japonicus, Medicago truncatula and Glycine max (Supplementary Fig. 10a). Phylogenetic analysis suggested both LjGSNORs from Lotus japonicus and MtGSNORs Medicago trucatula are most diverged from AtGSNOR (Supplementary Fig. 10b). This raised the question whether there is a subfunctionalization of GSNOR copies in species such as Lotus japonicus with GSNOR duplications. The gene structure and amino acid sequences of both LjGSNOR1 (Lj1g3v4528570) and LjGSNOR2 (Lj0g3v0071329) are still very conserved $(89.2 \%$ and $90.1 \%$ of identity respectively, both of them are predicted to be alcohol dehydrogenase class-3like clade) compared with AtGSNOR (Fig. 7a; Supplementary Fig. 10c). To test whether one or both homologs of LjGSNOR are involved in high $\mathrm{Fe}$ tolerance, we characterized L. japonicus knockout lines with LORE1 retrotransposon insertions ${ }^{40}$ in the exon of each of the two genes (Fig. 7a). We found that root growth of both Ljgsnor 1-1 and Ljgsnor 1-2 lines was more sensitive to high $\mathrm{Fe}$, while this sensitivity was not observed in Ljgsnor2-1 (Fig. 7d, e and Supplementary Fig. 11d). This increased root growth sensitivity was accompanied by a decreased size of the shoot system and increased accumulation of red pigments over time (Supplementary Fig. 11d). This showed that LjGSNOR1 is required for high Fe tolerance in Lotus japonicus and suggests that LjGSNOR2 has been subfunctionalized or is a homolog that has lost or is losing its function. A subfunctionalization is further supported by the absence of many phenotypes in Ljgsnor1 mutants that are present in Arabidopsis gsnor mutants ${ }^{28,29}$ that include visible development defects at both young stages (shorter roots under the normal condition) and mature stages (shorter stem, increased branching, reduced fertility and shorter siliques) (Supplementary Fig. 11e, f), as well as by the distinct, moderately anticorrelated $(\mathrm{r} \sim 0.2)$ expression pattern of the two LjGSNOR genes (Supplementary Fig. 12).

\section{Discussion}

As a major metal element in redox biology, Fe is required to induce radicals in many biological processes. However, this essential reactive property can lead to toxicity when Fe levels are too high. Our study identified GSNOR as underlying a major quantitative genetic locus controlling the natural variation of Arabidopsis tolerance to Fe toxicity. This is due to its central role in NO metabolism and subsequently in both nitrosative and oxidative stress regulation. Further, GSNOR is required for root tolerance to Fe toxicity not only in Arabidopsis but other dicots such as Lotus japonicus, and the monocot rice. Therefore, our findings not only shed light the underlying quantitative genetic and molecular mechanisms of plant adaptation to Fe-dependent 
a

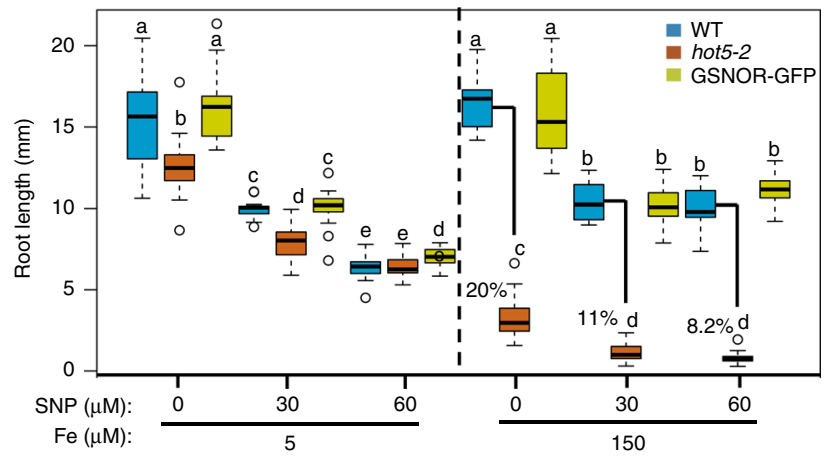

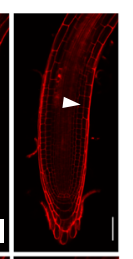
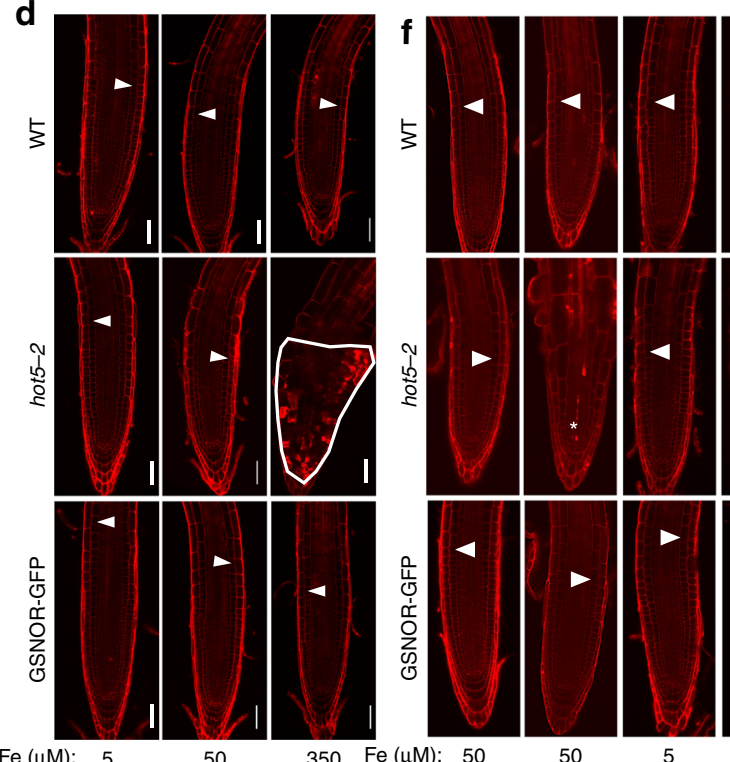

Fe $(\mu \mathrm{M}): 5$
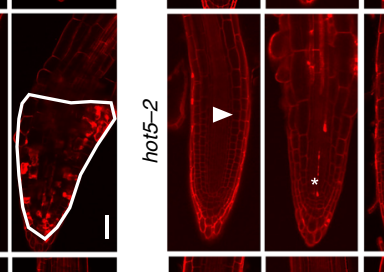

b

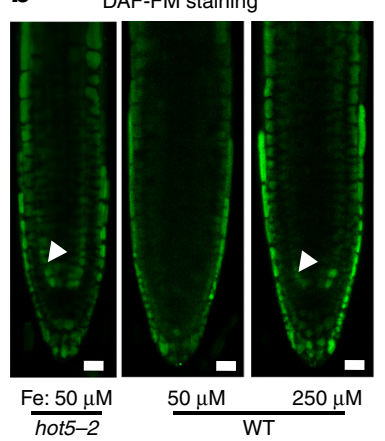

c

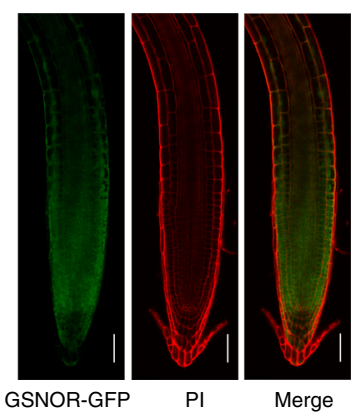

e

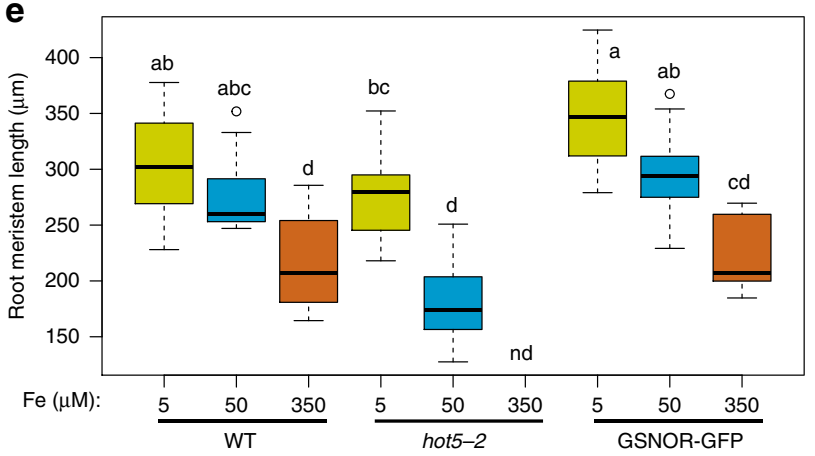

g

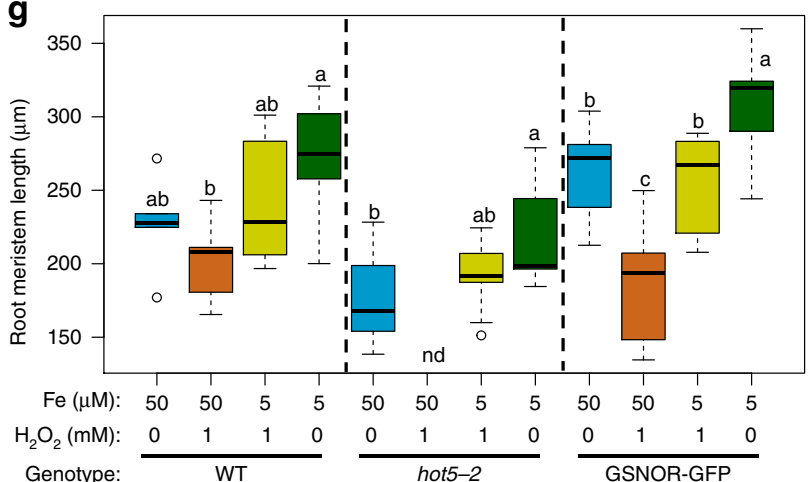

Fig. 6 GSNOR alleviates Fe-dependent $\mathrm{NO}$ and $\mathrm{H}_{2} \mathrm{O}_{2}$ toxicities to promote root meristem growth. a Box plots for root growth upon high Fe and nitric oxide (NO) donor (sodium nitroprusside, SNP) at day 7 ( $n=16$ biologically independent samples). Root length of hot5-2 relative to WT is shown in the right panel. b Fluorescence signal of root NO stained with DAF-FM at day 6 . White arrows denote cortex cells in meristems. c Fluorescence signal of root expressing pGSNOR:GSNOR-GFP at day 6 under control conditions. d Root meristems stained by propidium iodide (PI) at day 6 . e Box plots for root meristem of experiments depicted in (d) ( $n=10$ biologically independent samples). f Root meristems stained by PI upon $\mathrm{H}_{2} \mathrm{O}_{2}$ and Fe treatments at 7 days. White arrows indicate the border of elongation zone, while region outlined in white line and asterisk indicates cell death in the root meristem (d, $\mathbf{f}$ ). $\mathbf{g}$ Box plots for root meristem length of experiments depicted in (f) ( $n=9$ biologically independent samples). Different letters indicate the significant differences according to Tukey's HSD test $(p<0.05)$ between genotypes or different conditions in a given genotype. nd denotes not detected. Scale bar: $20 \mu \mathrm{m}$ in $(\mathbf{b})$; $50 \mu \mathrm{m}$ in (c, d and $\mathbf{f}$ ). The Source data of Fig. 6a, e, and g are provided in a Source Data file

toxicity, but also expose GSNOR as a prime target for genetic editing or marker-assisted breeding to develop crop varieties tolerant to Fe-dependent toxicity and help to increase rice productivity in regions with potential Fe toxicity particularly in West Africa. For this, additional studies need to be conducted in the relevant soils as $\mathrm{Fe}$ toxicity in plant not only depends on the $\mathrm{Fe}$ concentration in the soil, but is highly dependent on other soil parameters such as geochemistry and nutrient levels and on the particular genetic background.

While the amino acid sequence and the function of GSNOR for Fe toxicity tolerance is highly conserved at the interspecies level, we found a high diversity of regulatory SNPs in the promoter region and the introns of GSNOR within the Arabidopsis species. Complementation of the gsnor mutant with natural GSNOR variants indicated that polymorphisms that affect the expression of GSNOR contribute to the observed natural variation of root growth tolerance to high $\mathrm{Fe}$. Consistent with the importance of the expression level for its function in tolerance to high $\mathrm{Fe}$, modulating GSNOR expression levels in the mutant background was sufficient to increased Fe toxicity tolerance (Fig. 5; Supplementary Fig. 6). Interestingly, GSNOR expression is not induced by high Fe itself neither in Arabidopsis (Fig. 5c) nor in rice ${ }^{41,42}$, indicating that the base-line expression of GSNOR is important for its relevance for high Fe tolerance. The expression level effect is partly also supported by overexpression of 35 S:GSNOR in the Col-0 background (a high-Fe tolerant accession) that conferred a slightly more tolerance at $150 \mu \mathrm{M}$ Fe. However, there was no tolerance difference at higher levels of Fe (Fig. 3b). As in this line, 


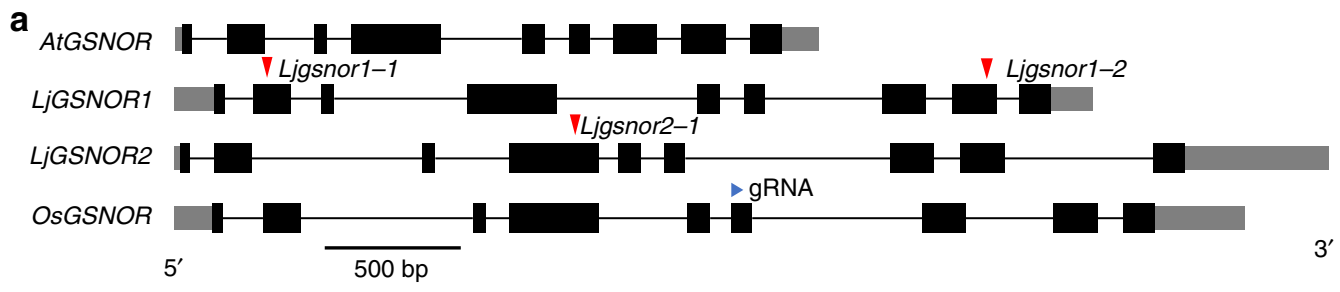

b
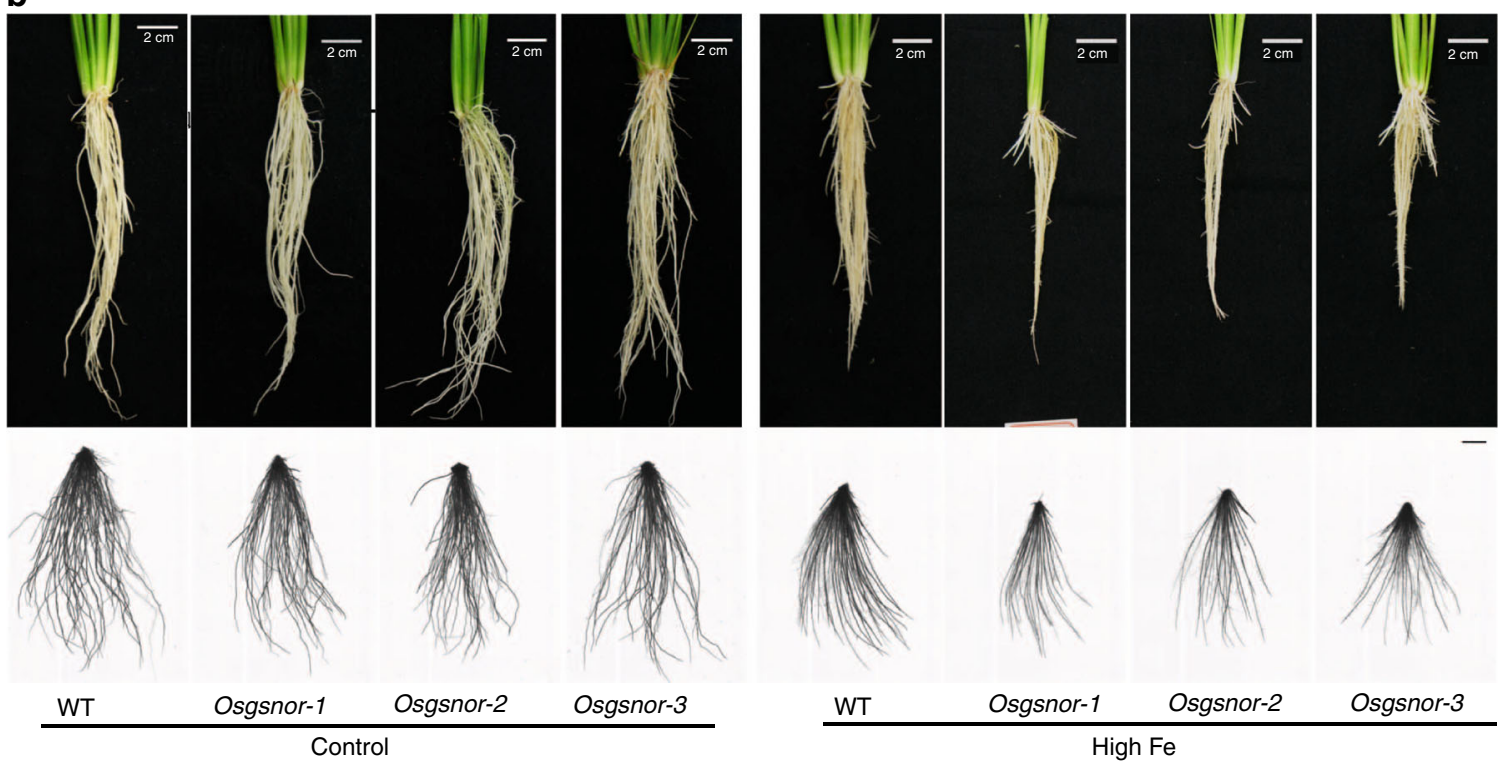

C

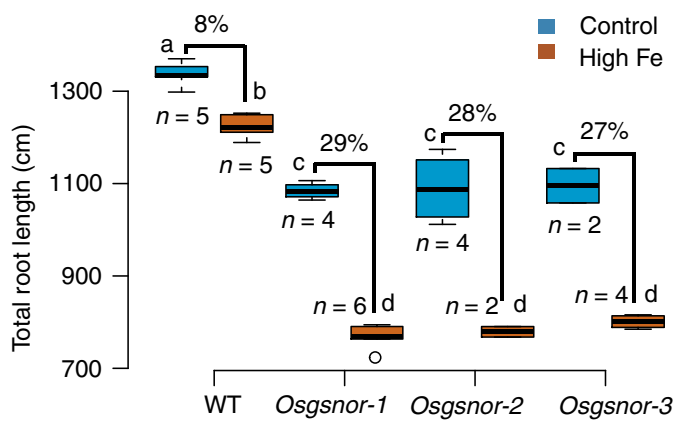

d
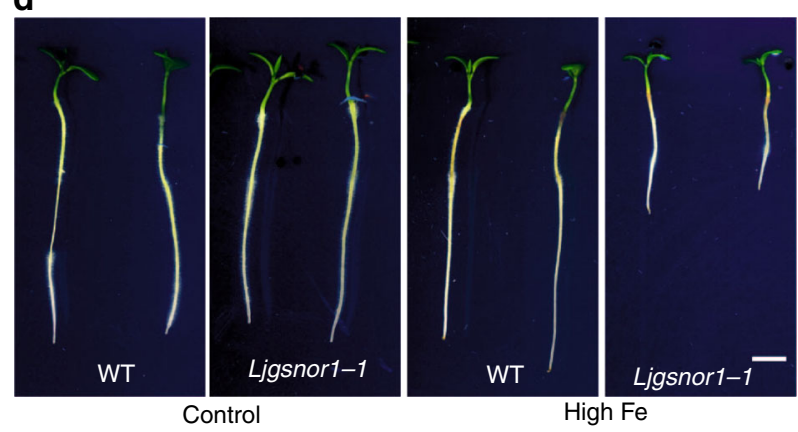

e
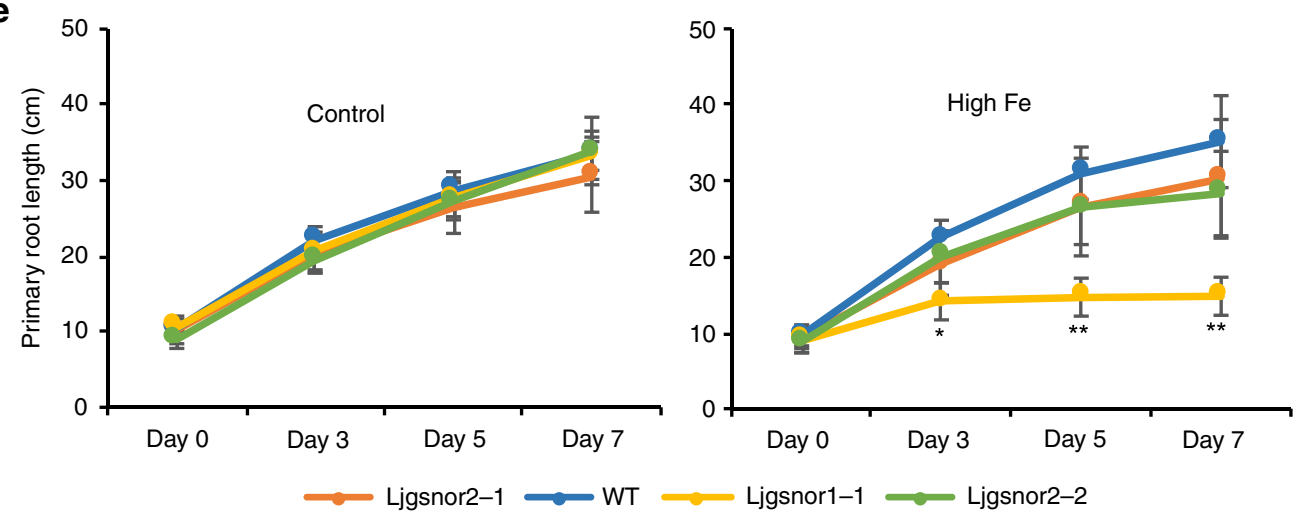

the $35 \mathrm{~S}$ promotor leads to a very high expression of GSNOR protein, as well as GSNOR activity ${ }^{43}$ (Supplementary Fig. 14), our results suggest that improved tolerance to Fe toxicity requires an optimal level of GSNOR (such as a level close to the GSNOR_Col-0 variant in the Ws-4 accession background) or a high GSNOR expression level in a specific cell type rather than in all cell types as conferred by the $35 S$ promoter.

While most of the data support the causality of GSNOR expression level variation for Fe tolerance, we cannot exclude the possibility that a nonsynonymous mutation (SNP:17684844) in 
Fig. 7 GSNOR function for Fe toxicity tolerance is conserved among higher plants. a Schematic representation of the gene structures and alleles of GSNOR from Lotus japonicus and rice. Red triangles indicate insert positions of LORE1 retrotransposon. Blue triangle indicates the position of guide RNA (gRNA) for CRISPR/Cas9. b, c Root growth of CRISPR/Cas9 knockout lines of OsGSNOR and WT. Upper panels, the roots before cutting; lower panels, the roots dispersed in water for total root length analysis. Scale bars: $2 \mathrm{~cm}$. Quantified total root length is shown in box plots c. N, biological replicates. The percentage indicates the difference caused by high Fe. Different letters indicated the significant differences by one-way ANOVA with Tukey's HSD test $(p<0.05)$. d Root growth at $50 \mu \mathrm{M} \mathrm{Fe}$ (control) and $350 \mu \mathrm{M} \mathrm{Fe}$ (high Fe) for 7 days. Scale bars: $5 \mathrm{~mm}$. e Mean primary root length. Error bars: standard deviation. $n=8$. Asterisk ${ }^{\star}$ and ${ }^{\star \star}$ indicate the significant difference between WT and mutant lines at $p<0.05$ and $p<0.01$, respectively (Student's $t$-test). The source data of Fig. 7c and e are provided in a Source Data file

GSNOR might also contribute to the phenotypic variation, even though it does not correlate with transcriptional variation of GSNOR. Nevertheless, high diversity (Supplementary Fig. 4) and strong linkage disequilibrium (Supplementary Fig. 3a, b) of the GSNOR related SNPs that we found make it difficult to distinguish the relative contribution of each SNP for the role of allelic variation for GSNOR function. It would be interesting to test the individual and combinatorial effects of these regulatory SNPs on GSNOR expression and on the binding affinity of upstream regulatory transcription factors.

Natural variants of GSNOR significantly contribute to $\mathrm{Fe}$ toxicity tolerance in Arabidopsis and it is likely to be caused by cis-regulatory variation. Rice also has large number of SNPs in the non-coding region of GSNOR (Supplementary Fig. 13). For example, we found 112 variations (primary allele frequency ranging $27 \%$ to $99.9 \%$ ) including SNPs and INDELs between 2.0 $\mathrm{kb}$ upstream and $1.0 \mathrm{~kb}$ downstream of this gene from 4729 accessions using RiceVarMap 2.0 (http://ricevarmap.ncpgr.cn/v2/ vars_in_gene/). Overall, these data suggest that GSNOR might be highly interesting for the development of Fe toxicity resilient crops. For this, it would be interesting to identify regulatory SNPs of rice GSNOR that could be directly used for gene editing and molecular marker-assisted selection for the breeding of Fe toxicity tolerance varieties. Similarly, non-coding SNPs of GSNOR were found to associate with the risk of childhood asthma in human ${ }^{44}$. These results suggest natural variation of GSNOR transcriptional levels may occur in both plants and animals.

Unlike the genes that confer hereditary diseases of Fe overload in humans and that are involved in Fe transport/homeostasis ${ }^{1}$, GSNOR seems to directly participate in the regulation of Feinduced redox-dependent cytotoxicity. Fe-catalyzed ROS production is thought to be the major reason for Fe toxicity ${ }^{14-16}$. However, studies in yeast and plant indicate that Fe-catalyzed ROS production may not account for Fe toxicity ${ }^{20,22}$, which suggests that other components (additional to ROS) might be also required for Fe-mediated toxicity. We demonstrated that GSNOR protects root meristem growth and prevents cell death caused by Fe-dependent $\mathrm{NO}$-induced nitrosative and $\mathrm{H}_{2} \mathrm{O}_{2}$-induced oxidative toxicity in Arabidopsis. These results revealed that NO is also required to generate $\mathrm{Fe}$-dependent redox toxicity, which advances our understanding of the toxic mechanisms of Fe. Additionally, NO-mediated potassium homeostasis can also participate in the inhibition on the root growth by high $\mathrm{Fe}^{45}$. Thus, as the interplay of high $\mathrm{Fe}$, ROS and reactive nitrogen species (RNS) is highly complex $^{46,47}$, it will be very interesting to investigate these interactions and their impact of cytotoxicity.

Several reports indicate that NO promotes Fe uptake under Fe deficiency conditions ${ }^{48,49}$, while $\mathrm{NO}$ almost has no effect on either the expression of the $\mathrm{Fe}$-acquisition genes or the ferric reductase activity at high level of $\mathrm{Fe}^{50}$. Since it has been reported that under control conditions three Fe deficiency responsive genes (bHLH100, 2.7-fold; bHLH39, 2.0-fold; bHLH38, 1.6-fold) were upregulated in the shoots of gsnor mutants compared to wild-type $^{29}$, we explored the possibility that accumulation of NO in the roots of the gsnor mutants might increase Fe transport and accumulation in high Fe conditions. We therefore measured the root expression of bHLH100, bHLH39, FIT1 all of which are key Fe deficiency induced transcription factors that directly activate the expression of ferric-chelate reductase FRO2 and the highaffinity ferrous Fe transporter IRT1 to increase Fe uptake and accumulation $^{51}$. Expression of none of these genes was different between gsnor mutant and the wild-type in response to high Fe treatment (Supplementary Fig. 15). The same held true for expression of FER1, which encodes for the major ferritin protein that binds and stores Fe in plants (Supplementary Fig. 15). This strongly suggests that Fe uptake and transport are not among the major factors for the high Fe susceptibility of the gsnor mutant.

Our results also expose $\mathrm{Fe}$ as an important factor in this interplay between ROS and RNS, for instance in inducing cell death. This goes in line with the increasingly recognized role of Fe as important initiator of cell death in a variety of animal systems ${ }^{17,18,52}$. Considering the GSNOR sequence and enzyme activity in GSNO reduction, which is highly conserved among bacteria, plants, and animals including humans ${ }^{28,29,31,32}$, and the fundamental relevance of NO signaling, GSNOR and the NO pathway may be promising targets to tackle high-Fe-dependent disease conditions ranging from Fe-toxicity in plants and $\mathrm{Fe}$ overload related conditions in humans.

\section{Methods}

Plant material and growth conditions. A total of 319 accessions of Arabidopsis thaliana were part of the Regional Mapping (RegMap) panel ${ }^{53}$. GSNOR knockout mutants hot5-2 and hot5-4 were in Col-0 and Ws- 4 background, respectively ${ }^{28}$ (gifts from Dr. Elizabeth Vierling, University of Massachusetts, USA). The proGSNOR:GSNOR-GFP was in hot5-2 background ${ }^{29}$. The GSNOR-overexpressing (GSNOR-OE) transgenic line ${ }^{30}$ and cue1- $6^{54}$ (gift from Dr. Ulf-Ingo Flügge, University of Cologne, Germany) were in Col- 0 background. The seeds of Ws- 4 were from the Versailles Arabidopsis Stock Center. The Lotus japonicus mutants (Gifu background) were ordered from Lotus Base (https://lotus.au.dk/) ${ }^{40}$ and were genotyped with the primers listed in Supplementary Table 2. Plant ID of Ljgsnor11, Ljgsnor1-2 and Ljgsnor2-1 are 30033535, 30060068, and 30075087 in Lotus base, respectively.

Arabidopsis thaliana seeds were sterilized using chlorine gas produced from the mixture of $130 \mathrm{ml} 10 \%$ sodium hypochlorite and $3.5 \mathrm{ml} 37 \%$ hydrochloric acid in a sealed box for $1 \mathrm{~h}$. Seeds that had been produced under uniform growth conditions were placed for $1 \mathrm{~h}$ in opened $1.5 \mathrm{ml}$ in the sterilization box, and then were stratified in water at $4{ }^{\circ} \mathrm{C}$ for 3 days in dark. The seeds were sown on the $1 \%$ agar plates and then these plates were vertically positioned in racks in a growth chamber under a 16/8-h light-dark cycle at $21^{\circ} \mathrm{C}$. When we tried to grow these hot5-2 and hot5-4 mutants on $1 \times \mathrm{MS}$ agar medium with $100 \mu \mathrm{M}$ Fe, the plants did not develop any roots (also see the previous study ${ }^{28}$ ). We therefore used $1 / 2 \times$ MS agar medium with $50 \mu \mathrm{M} \mathrm{Fe}$ and found that the roots of hot5-2 (which are slightly longer than hot5-4) reach around $60 \%$ of the wild-type length in this growth condition. We therefore used $1 / 2 \times$ MS agar medium from there-on for our further experiments except for the GWAS screening. If not specified differently, $1 / 2 \times$ MS agar medium contained $1 / 2 \times$ MS salts, $1 \%$ (w/v) sucrose, $1 \%(w / v)$ agar (Duchefa Biochemie) and was pH5.7. Different concentrations of Fe(III)-EDTA or other chemical compounds as were added as indicated in the text.

Seed sterilization of L. japonicus was conducted according to the protocol ${ }^{55}$ The seeds were scraped using sand paper for 1-2 min, then sterilized with $0.5 \%$ sodium hypochlorite and $0.1 \%$ Triton-X solution for $18 \mathrm{~min}$ and rinsed with sterile water 5-6 times. Seeds were germinated on moist, sterile paper on vertical plates in a growth chamber under a $16 / 8$-h light-dark cycle at $21^{\circ} \mathrm{C}$ for 3 days, then transferred to the agar plates with different treatment as indicated.

Rice seeds were sterilized with $10 \%$ sodium hypochlorite for $10 \mathrm{~min}$ and rinsed 5 times with sterile water. The sterile seeds were soaked in water at $28^{\circ} \mathrm{C}$ in dark for $3 \mathrm{~d}$, then were transferred to the modified Kimura B' solution for another $2 \mathrm{~d}$ 
under a cycle of $28^{\circ} \mathrm{C} / 16 \mathrm{~h}$ light and $25^{\circ} \mathrm{C} / 8 \mathrm{~h}$ dark, and with a light intensity of $400 \mu \mathrm{molm}^{-2} \mathrm{~s}^{-1}$ and $70 \%$ humidity. The modified Kimura B' solution contained $0.5 \mathrm{mM} \mathrm{MgSO}_{4} ; 0.36 \mathrm{mM} \mathrm{CaCl}_{2} ; 0.25 \mathrm{mM} \mathrm{KCl} ; 0.2 \mathrm{mM} \mathrm{NaH}_{2} \mathrm{PO}_{4} ; 50 \mu \mathrm{M} \mathrm{H}_{3} \mathrm{BO}_{3}$; $9 \mu \mathrm{M} \mathrm{MnCl}_{2} ; 0.3 \mu \mathrm{M} \mathrm{CuSO}_{4} ; 0.7 \mu \mathrm{M} \mathrm{ZnSO}_{4} ; 0.5 \mu \mathrm{M} \mathrm{Na}_{2} \mathrm{MoO}_{4}, 100 \mu \mathrm{M} \mathrm{Na}_{2} \mathrm{EDTA}-$ $\mathrm{Fe}$ (II),7.5 mM NH $\mathrm{Cl}_{4} \mathrm{Cl} \mathrm{mM} \mathrm{NaNO}_{3}$, and at $\mathrm{pH}$ 5.5. For high Fe treatment, the seedlings were grown in the modified Kimura B' solution with $1000 \mu \mathrm{M} \mathrm{Na}{ }_{2}$ EDTAFe (II) for another 2 weeks. The solution was exchanged every day.

Root phenotyping. To examine the specific effect of high Fe on the root growth, natural variation of root length among 319 Arabidopsis accessions at control $(1 \times \mathrm{MS}$ with $100 \mu \mathrm{M} F e$ (III) -EDTA) and high Fe (1× MS with $350 \mu \mathrm{M} \mathrm{Fe}$ (III) -EDTA) conditions was conducted in parallel. Each plate contained eight accessions with three biological replicates. To account for positional effects within and between the plates, we plated 12 seeds for each accession over four plates in a permutated block design. Images of these plates were acquired with $8 \mathrm{CCD}$ flatbed scanners (EPSON Perfection V600 Photo, Seiko Epson CO., Nagano, Japan) within half hour at the same time of at day3, day4, day5, day6, day7, day8, day10, and day13 after plating. Images were processed using the BRAT software ${ }^{56}$. Root length was measured using Fiji (http://fiji. $\mathrm{sc} / \mathrm{Fiji}$ ) in the experiments that included hot5 mutants and those with Lotus japonicus. If not specified differently, for Arabidopsis root length measurement, days after plating were indicated in the text or the figure legends. In rice, the number of lateral roots (root length longer than $5 \mathrm{~cm}$ ) was counted for each plant. For the total root length for each plant, the whole root system was cut at the junction between the shoot and the root and dispersed in water in a transparent tray. Images of these dispersed roots were acquired with a Dual Lens System scanner (Epson Perfection V700 PHOTO, China). The images were analyzed with WinRHIZO ${ }^{\mathrm{TM}} 2012$ (Zeal Quest Scientific Technology Co., Ltd. China).

Broad sense heritability calculation. All individuals of total root length in 319 natural accessions were used to calculate the broad-sense heritability $\left(\mathrm{H}_{2}=\mathrm{V}_{\mathrm{G}} /\right.$ $\left.\mathrm{V}_{\mathrm{P}}\right)$, which is defined as the proportion of phenotypic variation $\left(\mathrm{V}_{\mathrm{P}}\right)$ due to genetic variation $\left(\mathrm{V}_{\mathrm{G}}\right)$ (estimated from the between-line phenotypic variance).

Genome-wide association studies. Genome-wide association studies (GWAS) were performed for on the means of root length for each accession from 319 accessions at both the control and high Fe independently. In addition, GWAS was performed on the means of Fe tolerance index (\%), which was the ratio of root length under high $\mathrm{Fe}$ and root length under the control conditions. GWAS was conducted using $250 \mathrm{~K}$ SNPs from the RegMap panel in a mixed model algorithm that has been shown to correct for population structure confounding using the AMM method on the GWA-portal (https://gwas.gmi.oeaw.ac.at/) $)^{57}$. SNPs with minor allele counts greater or equal to 15 were considered for the analysis. The significance threshold for SNP associations was set at 5\% Bonferroni significance threshold to correct for multiple testing. A quantile-quantile (QQ) plot was generated and analyzed for each GWAS in GWA-portal. Linkage disequilibrium (LD) was visualized and analyzed using GWAPP (https://gwapp.gmi.oeaw.ac.at). Conditional (step-wise) GWAS ${ }^{58}$ was used to test whether SNPs associated independent of the primary associated SNP at a locus using GWAPP. With conditional GWAS analysis, the contribution of a locus for the observed phenotypic variation was also estimated using GWAPP. GWAS was performed in GWAPP/GWA-portal using default settings (no transformation and minor allele count $(\mathrm{MAC})>15$ ). The multi-trait mixed model (MTMM), which considers the within-trait and betweentrait variance components simultaneously for multiple trait ${ }^{59,60}$, was further used to generate multi-trait GWAS using the means of root length in control and high Fe conditions at day 10.

Determining haplotypes. A single significant GWAS peak was identified with root length variation in 319 Arabidopsis at high Fe (Fig. 1b). This GWAS peak contained 8 significant SNPs. By counting the unique combinations of these 8 SNPs in 319 accessions, 10 groups of haplotypes were defined (frequency of each haplotype occurring in more than 10 accessions).

Expression quantitative trait locus analysis. The data regarding the transcript levels of GSNOR in the shoots of 727 Arabidopsis accessions from 1001 genome project (https://www.ncbi.nlm.nih.gov/geo/query/acc.cgi?acc $=\mathrm{GSE} 80744{ }^{27}$ was extracted. 665 out of these 727 accessions were available in $1001 \mathrm{G} \mathrm{SNPs}^{61}$, and were used to perform a GWAS-based expression quantitative trait locus (eQTL) analysis with $1001 \mathrm{G} \mathrm{SNP}$ data set in GWA-portal (https://gwas.gmi.oeaw.ac.at/) using the GSNOR transcript level as a trait.

Construction of transgenic lines and CRISPR/Cas9 lines. For the comparison of GSNOR allele function, the 3115-bp genomic DNA sequence that encompassed $826 \mathrm{bp}$ the upstream of the translational start codon, the GSNOR gene and 166-bp downstream of the translational stop codon based on the Col-0 reference genome, was amplified from Col-0 (haplotype J of T-variant in the lead SNP) and Sf-2 (haplotype A of A-variant in the lead SNP), respectively. These DNA fragments were introduced into a modified pGreen0229 vector at the EcoR I site. This modified pGreen 0229 vector carried a Pro35S:PM-mCherry reporter gene, and kanamycin and basta resistance genes for bacteria and plants as a backbone, respectively ${ }^{62}$. The in-fusion cloning kit was used to construct all plasmids by following the user manual (Clontech, Japan). The fluorescence of mCherry reporter in the transformed seeds rather than antibiotic selection was used to select for positive transgenic lines using a fluorescence stereomicroscope (Leica MZ16 FA). Since the seed production is better in hot5-4 compared to hot5-2, all plasmids were verified by sequencing and transformed into the hot5-4 mutant (Ws-4 background) using agrobacterium-based floral dip method ${ }^{63}$. The cloning primers are listed in Supplementary Table 2.

All T1 seeds with mCherry signal were selected for further amplification. Based on the segregation of mCherry signals in seeds, all T2 lines (which derived from 4 (GSNOR_Col-0) or 10 (GSNOR_Sf2) independent T1 lines) rescued the root growth of hot5-4 to the Ws-4 similar level or higher level in control and high Fe conditions (Fig. 4b). Two independent T2 lines with a single insertion that displayed a similar pattern of root growth responses to high $\mathrm{Fe}$ as the average root growth response of all T2 lines of the same genotype were chosen to represent each construct of GSNOR_Col-0 and GSNOR_Sf-2 variants at the T3 generation. These representative T2 lines were selected to amplify the T3 generation. These homozygous T3 plants were used to analyze the phenotype and GSNOR expression.

For CRISPR/Cas9 knockout lines of OsGSNOR, a single guide RNA (Supplementary Table 2) binding to the coding sequence of OsGSNOR (LOC_Os02g57040) was designed using the CRISPRdirect tool (http://crispr.dbcls. $\mathrm{jp} /)^{64}$. The sgRNA were introduced into the CRISPR/Cas9 binary vector pBGK032 ${ }^{65}$. The $p B G K 032-O s G S N O R$ constructs were transformed into the calli of japonica rice cultivar Zhonghua 11 (ZH11) via Agrobacterium tumefaciens EHA105 using agrobacterium-mediated transformation ${ }^{66}$. The regenerated rice plants (T0) were selected by the hygromycin resistance and genotyped by sequencing using the primers (Supplementary Table 2). The biallelic, homozygous and heterozygous mutations in T0 plants were selected to amplify the seeds. The generation of OsGSNOR knockout lines was assisted by the Biogle Company (Hangzhou, China). The segregation of T1 seedlings were confirmed with genotyping and homozygous seedlings were used for phenotyping. Three independent homozygous lines with frameshifts: Osgsnor-1, Osgsnor-2 and Osgsnor-3 were chosen for phenotypic testing. They carry an insertion of ' $\mathrm{A}$ ' or ' $\mathrm{T}$ ' base at the position \#654 of coding sequence and a deletion between \#647 and \#659 of the coding sequence downstream of the ATG start codon, respectively.

Quantitative real time-PCR. Seedlings were grown on agar plates under control $(1 / 2 \times \mathrm{MS}$ with $50 \mu \mathrm{M} \mathrm{Fe})$ or high $\mathrm{Fe}(1 / 2 \times \mathrm{MS}$ with $350 \mu \mathrm{M} \mathrm{Fe})$ conditions for 6 days after germination. Whole roots were collected and immediately frozen in liquid nitrogen. Twenty-five seedlings of each genotype were used as one biological replicate. Total RNA was extracted using the RNeasy Mini kit (QIAGEN GmbH, Hilden, Germany). First strand cDNA was synthesized using RevertAid Reverse Transcriptase (Themo Fisher Scientific, USA). $2 \times$ SensiMix SYBR \& Fluorescein Kit (PEQLAB LLC, Wilmington, DE, USA) was used to prepare quantitative real time-PCR (qRT-PCR) reactions and PCR was performed in a Roche Lightcycler 96 (Roche) instrument. Three biological replicates and two technical replicates were analyzed for each gene. Relative quantifications were performed for all genes with the gene elongation factor 1 alpha (EF1a, AT5G60390) used as an internal reference. The primers used for qRT-PCR are shown in Supplementary Table 2.

Histochemical Fe staining with Perls/DAB stain. Fe accumulation in roots was assayed with Perls/DAB staining ${ }^{67}$. Briefly, 6-day-old seedlings were washed with 2 $\mathrm{mM} \mathrm{CaSO}_{4}$ and $10 \mathrm{mM}$ EDTA for $30 \mathrm{~min}$ and rinsed in water $5 \mathrm{~min}$, then incubated in $1 \mathrm{~mL}$ of $2 \%(\mathrm{v} / \mathrm{v}) \mathrm{HCl}$ and $2 \%(\mathrm{w} / \mathrm{v}) \mathrm{K}_{4}\left[\mathrm{Fe}(\mathrm{CN})_{6}\right]$ for $30 \mathrm{~min}$. These samples were then washed 3 times with water and incubated in $1 \mathrm{~mL}$ of $0.01 \mathrm{M} \mathrm{NaN}_{3}$ and $0.3 \% \mathrm{H}_{2} \mathrm{O}_{2}$ in methanol. After $30 \mathrm{~min}$, the samples were washed 3 times with $1 \mathrm{~mL}$ $0.1 \mathrm{M} \mathrm{KPO}_{4}$ buffer in $\mathrm{pH}$ 7.4. Then, the samples were incubated in $1 \mathrm{~mL}$ of 3,3'Diaminobenzidine tetrahydrochloride hydrate (DAB) solution $(0.025 \% \mathrm{DAB}$, $0.005 \% \mathrm{H}_{2} \mathrm{O}_{2}$ and $0.005 \% \mathrm{CoCl}_{2}$ in $0.1 \mathrm{M} \mathrm{KPO}_{4}$ in pH 7.4) in the dark. After 10 $\mathrm{min}$, the samples were washed at least 3 times with water. Finally, the Fe staining in roots was observed with an optical microscope.

Fresh shoot biomass measurement. Shoot tissues from four seedlings that grown on a single plate were collected as a biological replicate (one pool). Three biological replicates for each condition were analyzed. The shoot fresh weight per plant was calculated by dividing the weight of the pool by the number of plants.

GSNOR activity assay. GSNOR activity was measured by monitoring the decomposition of NADH at $340 \mathrm{~nm}^{32}$. Approximately 30 seedlings (8-day-old) were collected and weighted. The samples were ground and extracted with 4 volume $\left(4 \mu \mathrm{mg}^{-1}\right)$ of $50 \mathrm{mM}$ HEPES buffer $((20 \%$ glycerol, $10 \mathrm{mM} \mathrm{MgCl}, 1 \mathrm{mM}$ EDTA, $1 \mathrm{mM}$ EGTA, $1 \mathrm{mM}$ benzamidine, and $1 \mathrm{mM} \varepsilon$-aminocaproic acid, $\mathrm{pH} 8.0$ ), centrifuged to remove insoluble material at $4^{\circ} \mathrm{C}, 16,000 \mathrm{~g}$ for $15 \mathrm{~min}$. The supernatants were further clarified using Zeba ${ }^{\text {tw }}$ Spin Desalting Columns (ThermoFischer). The desalted fraction $(10 \mu \mathrm{L})$ was added to the $190 \mu \mathrm{l}$ assay mix $[20 \mathrm{mM}$ TRIS- $\mathrm{HCl}(\mathrm{pH} 8), 0.2 \mathrm{mM}$ NADH, and $0.5 \mathrm{mM}$ EDTA] with $400 \mu \mathrm{M}$ GSNO or $\mathrm{H}_{2} \mathrm{O}$, respectively. The $\mathrm{NADH}$ decomposition was monitored for $10 \mathrm{~min}$ after the addition of GSNO using a Tecan Safire II multi-mode plate reader. The rates were 
corrected for background NADH decomposition of each extract containing no GSNO. Another desalted fraction $(10 \mu \mathrm{L})$ was used for determining the total protein concentration by using Pierce ${ }^{\text {rit }}$ Rapid Gold BCA Protein Assay Kit (Thermo-Fischer) with this Tecan Safire II multi-mode plate reader. The corrected reduction rate of $\mathrm{NADH}$ was normalized against total protein amount. At least three biological replicates for each experiment were performed, the results of two independent experiments were similar, and the result of one independent experiment was presented in the figure.

Immunoblotting. Seven-day-old seedlings (around 30 plants) grown on $1 / 2 \mathrm{MS}$ medium or $1 / 2$ MS medium supplemented with $300 \mu \mathrm{M}$ Fe were harvested, weighed and ground in liquid nitrogen to fine powder. In total 5 volumes $\left(5 \mu \mathrm{l} . \mathrm{mg}^{-1}\right)$ of $2 \times$ NuPAGE lithium dodecyl sulfate (LDS) sample buffer (Thermo-Fischer) with Tris(2carboxyethyl) phosphine Hydrochloride (TCEP) was added to the samples and then boiled for $5 \mathrm{~min}$. After centrifugation at $15,000 \times g$ at $4{ }^{\circ} \mathrm{C}$ for $15 \mathrm{~min}, 15 \mu \mathrm{L}$ of supernatant were loaded, separated in a NuPAGE $4-12 \%$ Bis-Tris protein gel (Thermo-Fischer), followed by a wet transfer to nitrocellulose membrane (Bio-Rad). The GSNOR protein was detected by probing the membrane with anti-GSNOR antibody (AS09 647, Agrisera) at a dilution of 1:2000. Loading was controlled by using a rabbit anti-histone H3 antibody (9715 S, Cell Signaling Technology) at a dilution of 1:2000. Signal was detected using the SuperSignal ${ }^{\mathrm{m}}$ West Pico PLUS Chemiluminescent Substrate (Thermo-Fischer). At least two independent biological replicates (the samples from two different experiments) were performed for the western blot. The representative images from one experiment were presented.

Measurements of meristem size and cell length in roots. Six-day old Arabidopsis seedlings were stained with $15 \mu \mathrm{M}$ PI staining for $10 \mathrm{~min}$ and rinsed with water. The profile of root structure was visualized with PI staining and an LSM 700 confocal microscopy (Zeiss). The meristem size was measured from the quiescent center cells to first elongated cortex cell (median optical section was more than twice in length than in width). The cell length was measured from 30 fully elongated cortex cells in approximate 10 individual roots for each genotype in a given condition.

Nitric oxide staining of roots. 6-day old seedlings were incubated in $5 \mu \mathrm{M} 3$ amino, 4-aminomethyl-2,7-difluorofluorescein diacetate (DAF-FM DA, Molecular Probes, USA) in $10 \mathrm{mM}$ Tris- $\mathrm{HCl}$ with $\mathrm{pH} 7.5$ for $40 \mathrm{~min}$ at $25^{\circ} \mathrm{C}$ in the dark, and then were washed three times with fresh buffer $(10 \mathrm{mM}$ Tris- $\mathrm{HCl}, \mathrm{pH} 7.5)$ for 15 min. The fluorescence was observed with an LSM 700 confocal microscope.

Cell death staining. Cell death in the root tip was stained with $15 \mu \mathrm{M}$ PI (Sigma) or $250 \mathrm{nM}$ Sytox Orange nucleic acid Stain (Molecular Probes, Invitrogen) for 10 min, and rinsed twice with water before imaging with an LSM 700 confocal microscopy ${ }^{39}$.

Microscopy. All fluorescence was observed using an LSM 700 confocal microscopy (Zeiss). The excitation and emission wavelengths were set as follows: $488 \mathrm{~nm}$ and $485-545 \mathrm{~nm}$ for DAF-AM staining and GFP; $555 \mathrm{~nm}$ and $570-670 \mathrm{~nm}$ for PI and Sytox Orange staining. The images were analyzed with Fiji (http://fiji.sc/Fiji). For microscopy observations, at least 8 individual roots were analyzed for each genotype in a given condition in each independent experiment. At least 2 independent experiments were performed. The representative images from one experiment were presented.

Quantification and statistical analysis. All values are shown with box plots or as the means \pm standard deviation (SD) and the number (n) of samples is indicated in figures or figure legends. For box plots, the midline is the median of data; the upper and lower limits of the box are the third and first quartile, respectively; the whiskers are 1.5 times the interquartile range from the top (bottom) of the box to the furthest datum within that distance. No statistical methods were used to predetermine sample size. Significant differences between two samples for time-course experiments were determined with Student's $t$-test. Significant differences for multiple comparisons for single point experiment were determined by one-way or two-way ANOVA with Tukey's HSD test as indicated in figure legends. Statistical analyses and analysis of broad sense heritability were performed in the RStudio software (https://www.rstudio.com/).

Reporting Summary. Further information on research design is available in the Nature Research Reporting Summary linked to this article.

\section{Data availability}

Data supporting the findings of this work are available within the paper and its Supplementary Information files. A reporting summary for this Article is available as a Supplementary Information file. The datasets generated and analyzed during the current study are available from the corresponding authors upon request. The source data underlying Figs. 1a, 3b, 4b, 5a-d, 6a, e, g, 7c, e, and Supplementary Figs. 1a, 3c-f, 5, 6a-c, $7 \mathrm{~b}-\mathrm{d}, 8 \mathrm{~d}, 11 \mathrm{~b}, \mathrm{c}, 12,14$, and 15 , as well as Supplementary Table 1 are provided as a Source Data file.
Received: 30 January 2019 Accepted: 7 August 2019

Published online: 29 August 2019

\section{References}

1. Sheftel, A. D., Mason, A. B. \& Ponka, P. The long history of iron in the universe and in health and disease. Biochim. Biophys. Acta 1820, 161-187 (2012).

2. Ponnamperuma, F. N., Bradfield, J. F. \& Peech, M. Physiological disease of rice attributable to iron toxicity. Nature 175, 275 (1955).

3. Vose, P. B. Iron nutrition in plants: a world overview. J. Plant Nutr. 5, 233-249 (1982).

4. Audebert, A. \& Fofana, M. Rice yield gap due to iron toxicity in West Africa. J. Agron. Crop Sci. 195, 66-76 (2009).

5. Chérif, M., Audebert, A., Fofana, M. \& Zouzou, M. Evaluation of iron toxicity on lowland irrigated rice in West Africa. Tropicultura 27, 88-92 (2009).

6. Becker, M. \& Asch, F. Iron toxicity in rice-conditions and management concepts. J. Soil Sci. Plant Nutr. 168, 558-573 (2005).

7. Haefele, S. M., Nelson, A. \& Hijmans, R. J. Soil quality and constraints in global rice production. Geoderma 235-236, 250-259 (2014).

8. Sikirou, M. et al. Genetic improvement of iron toxicity tolerance in riceprogress, challenges and prospects in West Africa. Plant Prod. Sci. 18, 423-434 (2015).

9. Matthus, E. et al. Loci, genes, and mechanisms associated with tolerance to ferrous iron toxicity in rice (Oryza sativa L.). Theor. Appl. Genet. 128, 2085-2098 (2015).

10. Liu, H. et al. QTL underlying iron and zinc toxicity tolerances at seedling stage revealed by two sets of reciprocal introgression populations of rice (Oryza sativa L). Crop J. 4, 280-289 (2016).

11. Nugraha, Y., Wahyuning, S., Ghulamahdi, M., Suwarno, S. \& Aswidinnoor, H. Markers-traits association for iron toxicity tolerance in selected Indonesian rice varieties. J. Biodiversitas 17, 753-763 (2016).

12. Meng, L. et al. Association mapping of ferrous, zinc, and aluminum tolerance at the seedling stage in Indica Rice using MAGIC populations. Front. Plant Sci. 8, 1822 (2017).

13. Zhang, J. et al. QTL mapping and candidate gene analysis of ferrous iron and zinc toxicity tolerance at seedling stage in rice by genome-wide association study. BMC Genom. 18, 828 (2017).

14. Toyokuni, S. Iron-induced carcinogenesis: the role of redox regulation. Free Radic. Biol. Med. 20, 553-566 (1996).

15. Becana, M., Moran, J. F. \& Iturbe-Ormaetxe, I. Iron-dependent oxygen free radical generation in plants subjected to environmental stress: toxicity and antioxidant protection. Plant Soil 201, 137-147 (1998).

16. Dixon, S. J. \& Stockwell, B. R. The role of iron and reactive oxygen species in cell death. Nat. Chem. Biol. 10, 9-17 (2014).

17. Dixon, S. J. et al. Ferroptosis: an iron-dependent form of nonapoptotic cell death. Cell 149, 1060-1072 (2012).

18. Mai, T. T. et al. Salinomycin kills cancer stem cells by sequestering iron in lysosomes. Nat. Chem. 9, 1025-1033 (2017).

19. Müller, J. et al. Iron-dependent callose deposition adjusts root meristem maintenance to phosphate availability. Dev. Cell 33, 216-230 (2015)

20. Lin, H., Li, L., Jia, X., Ward, D. M. \& Kaplan, J. Genetic and biochemical analysis of high iron toxicity in yeast: iron toxicity is due to the accumulation of cytosolic iron and occurs under both aerobic and anaerobic conditions. $J$. Biol. Chem. 286, 3851-3862 (2011).

21. Tsukagoshi, H., Busch, W. \& Benfey, P. N. Transcriptional regulation of ROS controls transition from proliferation to differentiation in the root. Cell 143, 606-616 (2010).

22. Reyt, G., Boudouf, S., Boucherez, J., Gaymard, F. \& Briat, J. F. Iron and ferritin dependent ROS distribution impact Arabidopsis root system architecture. Mol. Plant 8, 439-453 (2015).

23. Ravet, K. et al. Ferritins control interaction between iron homeostasis and oxidative stress in Arabidopsis. Plant J. 57, 400-412 (2009).

24. Briat, J. F., Duc, C., Ravet, K. \& Gaymard, F. Ferritins and iron storage in plants. Biochim. Biophys. Acta 1800, 806-814 (2010).

25. Wilkins, D. A. The measurement of tolerance to edaphic factors by means of root growth. New Phytol. 80, 623-633 (1978).

26. Li, G., Xu, W., Kronzucker, H. J. \& Shi, W. M. Ethylene is critical to the maintenance of primary root growth and Fe homeostasis under Fe stress in Arabidopsis. J. Exp. Bot. 66, 2041-2054 (2015).

27. Kawakatsu, T. et al. Epigenomic diversity in a global collection of Arabidopsis thaliana accessions. Cell 166, 492-505 (2016).

28. Lee, U., Wie, C., Fernandez, B. O., Feelisch, M. \& Vierling, E. Modulation of nitrosative stress by $\mathrm{S}$-nitrosoglutathione reductase is critical for thermotolerance and plant growth in Arabidopsis. Plant Cell 20, 786-802 (2008).

29. Xu, S., Guerra, D., Lee, U. \& Vierling, E. S-nitrosoglutathione reductases are lowcopy number, cysteine-rich proteins in plants that control multiple developmental and defense responses in Arabidopsis. Front. Plant Sci. 4, 430 (2013). 
30. Achkor, H. et al. Enhanced formaldehyde detoxification by overexpression of glutathione-dependent formaldehyde dehydrogenase from Arabidopsis. Plant Physiol. 132, 2248-2255 (2003).

31. Liu, L. et al. A metabolic enzyme for S-nitrosothiol conserved from bacteria to humans. Nature 410, 490-494 (2001).

32. Sakamoto, A., Ueda, M. \& Morikawa, H. Arabidopsis glutathione-dependent formaldehyde dehydrogenase is an S-nitrosoglutathione reductase. FEBS Lett. 515, 20-24 (2002).

33. Feechan, A. et al. A central role for S-nitrosothiols in plant disease resistance. Proc. Natl Acad. Sci. USA 102, 8054-8059 (2005).

34. Chen, R. et al. The Arabidopsis PARAQUAT RESISTANT2 gene encodes an Snitrosoglutathione reductase that is a key regulator of cell death. Cell Res. 19, 1377-1387 (2009).

35. Kovacs, I. et al. ROS-mediated inhibition of $S$-nitrosoglutathione reductase contributes to the activation of anti-oxidative mechanisms. Front. Plant Sci. 7, 1669 (2016).

36. He et al. Nitric oxide represses the Arabidopsis floral transition. Science 305, 1968-1971 (2004).

37. Fernández-Marcos, M., Sanz, L., Lewis, D. R., Muday, G. K. \& Lorenzo, O. Nitric oxide causes root apical meristem defects and growth inhibition while reducing PIN-FORMED 1 (PIN1)-dependent acropetal auxin transport. Proc. Natl Acad. Sci. USA 108, 18506-18511 (2011).

38. Dinis-Oliveira, R. J. et al. Paraquat poisonings: mechanisms of lung toxicity, clinical features, and treatment. Crit. Rev. Toxicol. 38, 13-71 (2008).

39. Truernit, E. \& Haseloff, J. A simple way to identify non-viable cells within living plant tissue using confocal microscopy. Plant Methods 4, 15 (2008).

40. Małolepszy, A. et al. The LORE1 insertion mutant resource. Plant J. 88, 306-317 (2016).

41. Quinet., M. et al. Combined transcriptomic and physiological approaches reveal strong differences between short- and long-term response of rice (Oryza sativa) to iron toxicity. Plant Cell Environ. 35, 1837-1859 (2012).

42. Bashir, K. et al. Transcriptomic analysis of rice in response to iron deficiency and excess. Rice 7, 1-15 (2014)

43. Rustérucci, C., Espunya, M. C., Díaz, M., Chabannes, M. \& Martínez, M. C. Snitrosoglutathione reductase affords protection against pathogens in Arabidopsis, both locally and systemically. Plant Physiol. 143, 1282-1292 (2007).

44. $\mathrm{Wu}, \mathrm{H}$. et al. Genetic variation in S-nitrosoglutathione reductase (GSNOR) and childhood asthma. J. Allergy Clin. Immunol. 120, 322-328 (2007).

45. Zhang, L. et al. Excess iron stress reduces root tip zone growth through nitric oxide-mediated repression of potassium homeostasis in Arabidopsis. New Phytol. 219, 259-274 (2018).

46. Pacelli, R. et al. Nitric oxide potentiates hydrogen peroxide-induced killing of Escherichia coli. J. Exp. Med. 182, 1469-1479 (1995).

47. Bosworth, C. A., Toledo, J. C. Jr., Zmijewski, J. W., Li, Q. \& Lancaster, J. R. Jr. Dinitrosyliron complexes and the mechanism(s) of cellular protein nitrosothiol formation from nitric oxide. Proc. Natl Acad. Sci. USA. 106, 4671-4676 (2009).

48. Graziano, M. \& Lamattina, L. Nitric oxide accumulation is required for molecular and physiological responses to iron deficiency in tomato roots. Plant J. 52, 949-960 (2007).

49. Chen, W. W. et al. Nitric oxide acts downstream of auxin to trigger root ferricchelate reductase activity in response to iron deficiency in Arabidopsis. Plant Physiol. 154, 810-819 (2010)

50. García, M. J., Suárez, V., Romera, F. J., Alcántara, E. \& Pérez-Vicente, R. A new model involving ethylene, nitric oxide and Fe to explain the regulation of Fe-acquisition genes in Strategy I plants. Plant Physiol. Biochem. 49, 537-544 (2011).

51. Wang, N. et al. Requirement and functional redundancy of Ib subgroup bHLH proteins for iron deficiency responses and uptake in Arabidopsis thaliana. Mol. Plant 6, 503-513 (2013).

52. Ferris, C. D. et al. Haem oxygenase-1 prevents cell death by regulating cellular iron. Nat. Cell Biol. 1, 152-157 (1999).

53. Horton, M. W. et al. Genome-wide patterns of genetic variation in worldwide Arabidopsis thaliana accessions from the RegMap panel. Nat. Genet. 44, 212-216 (2012).

54. Voll, L. et al. The phenotype of the Arabidopsis cue1 mutant is not simply caused by a general restriction of the shikimate pathway. Plant J. 36, 301-317 (2003).

55. Giovannetti, M., Małolepszy, A., Göschl, C. \& Busch, W. Large-Scale Phenotyping of Root Traits in the Model Legume Lotus japonicus. In: Busch W. (eds) Plant Genomics. Methods in Molecular Biology, vol 1610. Humana Press, New York, NY (2017).

56. Slovak, R. et al. A scalable open-source pipeline for large-scale root phenotyping of Arabidopsis. Plant Cell 26, 2390-2403 (2014).

57. Seren, Ü. et al. GWAPP: a web application for genome-wide association mapping in Arabidopsis. Plant Cell 24, 4793-4805 (2012).

58. Yang, J. et al. Conditional and joint multiple-SNP analysis of GWAS summary statistics identifies additional variants influencing complex traits. Nat. Genet. 44, 369-375 (2012). S1-3.
59. Korte, A. et al. A mixed-model approach for genome-wide association studies of correlated traits in structured populations. Nat. Genet. 44, 1066-1071 (2012).

60. Lippert, C., Casale, F. P., Rakitsch, B. \& Stegle, O. LIMIX: genetic analysis of multiple traits. Preprint at http://biorxiv.org/content/early/2014/05/21/003905 (2014).

61. 1001 Genomes Consortium. 1,135 Genomes Reveal the Global Pattern of Polymorphism in Arabidopsis thaliana. Cell 166, 481-491 (2016).

62. Satbhai, S. B. et al. Natural allelic variation of FRO2 modulates Arabidopsis root growth under iron deficiency. Nat. Commun. 8, 15603 (2017).

63. Clough, S. J. \& Bent, A. F. Floral dip: a simplified method for Argobacteriummediated transformation of Arabidopsis thaliana. Plant J. 16, 735-743 (1998).

64. Naito, Y., Hino, K., Bono, H. \& Ui-Tei, K. CRISPRdirect: software for designing CRISPR/Cas guide RNA with reduced off-target sites. Bioinformatics 31, 1120-1123 (2015).

65. Li, W. et al. A natural allele of a transcription factor in rice confers broadspectrum blast resistance. Cell 170, 114-126 (2017).

66. Nishimura, A., Aichi, I. \& Matsuoka, M. A protocol for Agrobacteriummediated transformation in rice. Nat. Protoc. 1, 2796-2802 (2006).

67. Roschzttardtz, H., Conejero, G., Curie, C. \& Mari, S. Identification of the endodermal vacuole as the iron storage compartment in the Arabidopsis embryo. Plant Physiol. 151, 1329-1338 (2009).

\section{Acknowledgements}

We thank Samantha Krasnodebski, Anna Malolepszy and Marco Giovannetti for assistance with the experiments in Lotus japonicus; Hironaka Tsukagoshi and Hatem Rouached for critical reading of the manuscript; Santosh B. Satbhai, Takehiko Ogura, Marco Giovannetti, and other members from the Busch lab for valuable discussions and comments; Eriko Sasaki and Ümit Seren for assistance with the GWAS analysis; Elizabeth Vierling (University of Massachusetts), Ulf-Ingo Flügge (University of Cologne) and Lotus Base for sharing seeds. This work was supported by the Lise-Meitner program of Austrian Science Fund (FWF) (grant number M1826 Meitner-Programm to B.L.), by the National Institute of General Medical Sciences of the National Institutes of Health (grant number R01GM127759 to W.B.), by the key project of National Natural Science Foundation of China (grant number 31430095 to W.S.), and by funds from the Austrian Academy of Sciences through the Gregor Mendel Institute and start-up funds from the Salk Institute for Biological Studies.

\section{Author contributions}

B.L. and W.B. conceived and designed the experiments; B.L. and J.H. performed experiments on the Arabidopsis thaliana and Lotus japonicus; B.L. and L.S. performed experiments on rice; B.L., W.B., W.S. and J.C. provided reagents; B.L., W.B., C.G., L.S. and J.H. analyzed data; B.L. and W.B. wrote the manuscript which was revised and approved by all authors.

\section{Additional information}

Supplementary Information accompanies this paper at https://doi.org/10.1038/s41467 019-11892-5.

Competing interests: The authors declare no competing interests.

Reprints and permission information is available online at http://npg.nature.com/ reprintsandpermissions/

Peer review information: Nature Communications thanks Petra Bauer, Jianru Zuo, and the other anonymous reviewer(s) for their contribution to the peer review of this work. Peer reviewer reports are available.

Publisher's note: Springer Nature remains neutral with regard to jurisdictional claims in published maps and institutional affiliations.

Open Access This article is licensed under a Creative Commons Attribution 4.0 International License, which permits use, sharing, adaptation, distribution and reproduction in any medium or format, as long as you give appropriate credit to the original author(s) and the source, provide a link to the Creative Commons license, and indicate if changes were made. The images or other third party material in this article are included in the article's Creative Commons license, unless indicated otherwise in a credit line to the material. If material is not included in the article's Creative Commons license and your intended use is not permitted by statutory regulation or exceeds the permitted use, you will need to obtain permission directly from the copyright holder. To view a copy of this license, visit http://creativecommons.org/ licenses/by/4.0/

(C) The Author(s) 2019 